\title{
TWO-STAGE BASED DYNAMIC EARTH-ROCK TRANSPORTATION ASSIGNMENT PROBLEM UNDER FUZZY RANDOM ENVIRONMENT TO EARTH-ROCK DAM CONSTRUCTION
}

\author{
Jiuping XU', b , Cuiying FENG ${ }^{\mathrm{b}}$ \\ ${ }^{a}$ State Key Laboratory of Hydraulics and Mountain River Engineering, Sichuan University, \\ Chengdu, 610064, P. R. China \\ ${ }^{b}$ Uncertainty Decision-Making Laboratory, Sichuan University, Chengdu, 610064, P. R. China
}

Received 26 Sep 2012; accepted 23 Jan 2013

\begin{abstract}
This paper discusses a two-stage based dynamic transportation assignment problem (TS-based DTAP) under a fuzzy random environment in an earth-rock transportation system. This problem is a multi-objective dynamic programming optimization process for minimizing total operational cost, transportation duration and total waste. Triangular fuzzy random numbers are used for the uncertain parameters, and a hybrid crisp approach and an expected value operator are introduced to deal with these uncertainties. A dynamic programming based contraction particle swarm optimization is developed to solve the proposed expected value model for TS-based DTAP. Then, the earth-rock dam construction at Pubugou Hydropower project is used as a practical application to verify the proposed approach. Results and analysis are presented to highlight the performance of the proposed TS-based DTAP model and the optimization method, which proves to be effective and relatively efficient compared to the models under other environments and a standard PSO algorithm.
\end{abstract}

Keywords: dynamic transportation assignment problem, multi-objective optimization, fuzzy random environment, contraction particle swarm optimization.

\section{Introduction}

As the special case of minimum cost network flow problem, assignment problem plays a very important role in many real-world applications, such as $\mathrm{Li}$ and Simha (2000), Clementi et al. (2003) and Bliemer and Bovy (2003). In large scale hydropower construction projects, transportation assignment problem solutions are crucial and must be dealt with urgently because of high cost, long construction durations and other important reasons. Further, the optimization of transportation assignment problems requires combinatorial optimization, combining transportation costs, duration and waste optimization, which in turn have a significant influence on each other. This is reflected in real conditions, where there is increasing pressure to shorter transportation duration to reduce or eliminate extra project expenses by ensuring the early arrival of materials, a shortening of the construction project completion time and the improvement of construction efficiency. With this in mind, this paper focuses on a multi-objective optimization of a two-stage based earth-rock dam construction transportation system, with the aim of minimizing total operational cost, transportation duration and total waste.
Dynamic transportation assignment problems (DTAP) are often encountered in many systems, such as urban planning traffic networks, closed traffic network systems, field service support systems, container transportation and flowshop-type production systems (Crainic et al. 2004; Wang et al. 2008; Ebben et al. 2004; Papadopoulos 1996; De Meo et al. 2008; Celik et al. 2009; Anghinolfi, Paolucci 2009; Hurink, Knust 2001). Linear programming, bi-level programming and multi-objective programming are usually used to solve such transportation problems, as in Luathep et al. (2011), Ali and Sik (2012), Maher et al. (2001), Cao et al. (2007), Islam and Roy (2006), El-Wahed and Lee (2006). While these studies have made a significant contribution to DTAP and have solved many practical problems, they rarely discuss double multistage transportation systems, and do not take possible waste into consideration. In particular, these studies have not considered multi-objective optimization and multistage optimization together, nor discussed problems from a dynamic view for large scale hydropower construction projects. By considering the characteristics of different areas (borrow areas, stockpile areas and filling areas), introducing a double multistage transportation system, and including the different objectives in a

Corresponding author: Jiuping $\mathrm{Xu}$

E-mail:xujiuping@scu.edu.cn 
dynamic environment, decision-makers are able to dynamically allocate resources so that the total operational cost, transportation duration and total waste are minimized. Specifically, several kinds of transportation carriers are proposed. Above all, a two-stage based dynamic transportation assignment problem (TS-based DTAP) using a multi-objective dynamic programming process for large scale hydropower projects is proposed in this paper.

Transportation systems are often complex with inevitably encountering uncertain parameters. Though previous research (Cascetta 1989; Barbarosoglu, Arda 2004; Shu et al. 2005; Watling 2006) has successfully used probability theory to solve many transportation problems, sometimes the probability distributions for uncertain parameters may be unknown or just partially known because of a lack of statistical data. In this case, random variables are not able to adequately describe these uncertain parameters, and fuzzy set theory is a more appropriate choice. First proposed by Zadeh (1965), and consequently developed by researchers such as Dubois and Prade (1978), fuzzy theory has been a useful tool in dealing with ambiguous information, and has been applied to forecasting (Möller, Reuter 2007), decision-making (Pardo, de la Fuente 2007), and control of actions in environments which are characterized by vagueness, imprecision, and subjectivity (Bojadziev, G., Bojadziev, M. 1997; Sumalee et al. 2006). For instance, if experienced experts are asked about the unit transportation cost between two places, the answer is more likely to be around a certain value rather than an absolute value because of possibility of unforeseen factors such as changing weather, labor inefficiency, or the road and traffic condition. Since this information is described using linguistic terms, conventional probability is not suitable to model this imprecise content. So in this case, unit transportation cost could be expressed as a fuzzy variable rather than as a constant, as outlined in Chanas et al. (1984), Chanas and Kuchta (1996), Teodorovic (1999), Liu and Kao (2004), and Peidro et al. (2009). There have also been studies considering fuzzy demand, purchase cost and holding cost in inventory control systems (Xu, Liu 2008). While these studies have significantly improved the uncertainty in transportation system management, they are incapable of reflecting the multiple uncertainties of parameters with combinations of both fuzziness and randomness. In practical large scale construction projects, imprecision and complexity cannot be dealt with using simple fuzzy logic or random logic, and TS-based DTAP control often takes place in a hybrid uncertain environment. In this case, fuzzy random variables, introduced by Kwakernaak $(1978,1979)$, Puri and Ralescu (1986), Kruse and Meyer (1987), and used in modeling and analyzing "imprecise values" which is associated with the sample space of a random experiment using fuzzy-set functions (Gil et al. 2006), can be employed. This approach has received approval from many scholars and encouraged further research in uncertain events. Dutta et al. (2005) constructed a single-period inventory model with fuzzy random variable demand.
Ammar (2008) presented fuzzy random multi-objective quadratic programming solutions for application in portfolio problems. Xu and Liu (2011) and Xu et al. (2012c) developed a facility location-allocation problem and a resources allocation problem in a mixed random and fuzzy environment. These studies show how efficient fuzzy random variables can be when dealing with hybrid uncertain environments where fuzziness and randomness co-exist. However, the application of fuzzy random variables has not yet been applied to the TS-based DTAP to solve practical problems. In this paper, fuzzy random uncertainty is adopted to describe the characteristics of the hybrid uncertain environment for TS-based DTAP, and the results and analysis obtained in section 4.4.4 "Model Comparison in Different Environments" are presented to highlight the superiority of usage for the fuzzy random variables.

With the TS-based DTAP being intrinsically difficult and the model being nonlinear, non-convex and non-differentiable, it follows that the search for exact algorithms is futile. Effort has been made to develop effective heuristic algorithms to solve the TS-based DTAP. Over the past three decades, many efficient metaheuristic search techniques have been developed, such as genetic algorithms (Gen et al. 1998), ant algorithms (Doerner et al. 2001; Bell, McMullen 2004; Vitins, Axhausen 2009), simulated annealing (Naderi et al. 2009; Friesz et al. 1993) and the tabu search. Recently, a new population-based stochastic search method, particle swarm optimization (PSO), inspired by the social behaviors of animals like fish schooling and bird flocking, was proposed for optimization (Kennedy, Eberhart 1995). Existing publications indicate that the PSO method has comparable or even superior performance when solving many NP-hard problems and has a fast and stable convergence (Clerc, Kennedy 2002). Because it is easy-to-implement, efficient and effective, PSO is adopted in this study to develop a dynamic programming based contraction particle swarm optimization (DP-based CPSO) algorithm for solving the transformed optimization model.

However, there is still no research which takes the multi-objective, double multi-stage, dynamic, and hybrid uncertainty factors into consideration comprehensively. So in this paper, the following techniques are used: first, this paper, in reference to the earth-rock transportation system at the Pubugou Hydropower Project, establishes a multi-objective dynamic optimal control model to solve the TS-based DTAP under a fuzzy random environment. Second, the description of the TS-based DTAP under a fuzzy random environment is described. Because the TS-based DTAP control in a large scale construction project takes place in a hybrid uncertain environment, source data was first gathered from the engineers, then estimated using maximum likelihood method, finally the expected values of which were determined using a hybrid crisp approach and an expected value operator. Another technique used here is the DP-based CPSO, which was developed based on the particular nature of the model to 
solve the above problem, by automatically controlling the particle-updating in the feasible solution space to find the optimal solution. The final contribution is to illustrate the application of the optimization method on actual data collected from the Pubugou Hydropower Project, which demonstrates the value of practical application of the optimization method to a construction transportation system.

The rest of this paper is organized as follows: Section 1 describes the key problem statement for TS-based DTAP. A TS-based DTAP model with fuzzy random coefficients is then formulated to minimize the total operational cost, transportation duration and total waste in Section 2. In Section 3, a DP-based CPSO algorithm is developed to solve the model. In Section 4, the earth-rock dam construction at the Pubugou Hydropower Project is used as a practical application to demonstrate the practicality of the modeling method and the efficacy of the developed algorithm. Finally, concluding remarks are given in the last section.

\section{Key problem statement}

\subsection{Multistage earth-rock dam construction project with two-stage based transportation system}

In a large scale hydropower construction project, earthrock dam construction is a crucial task. The complete earth-rock dam construction process in this paper can be divided into three stages on the basis of total construction duration, construction sequence and construction diversion. The three stages in the order of earth-rock dam construction are foundation filling, central dam filling and upper dam filling as shown in Figure 1, and each stage requires a different quantity of earth-rock depending on the construction strength, area and features. The main task of the first stage is foundation filling until a solid foundation is reached. During the second stage, the main task is central dam filling and cofferdam filling, and this is the most crucial period for the entire earth-rock dam construction, which requires intense efforts. The main task of the third stage is the filling of the dam crest, and construction of the dam's corresponding height, thickness and slope protection construction, all of which are dependent on variations in the water level in the upper dam area.

From the construction site's environmental features and the characteristics of the transportation routes, the earth-rock dam construction project transportation system can be divided into two stages. The first stage is the transportation from the borrow areas to the stockpile areas using dump trucks, and the second stage is from the stockpile areas to their corresponding filling areas using belt conveyors, the details of which are in Figure 1. For each transportation route, the transportation distance, road condition, transportation intensity and other objective factors are quite dissimilar, meaning that the various parameters are also different and uncertain. To improve operational efficiency and reduce traffic accidents, each dump truck driver is allocated a fixed transportation route. Therefore, the transportation routes selection and the earth-rock

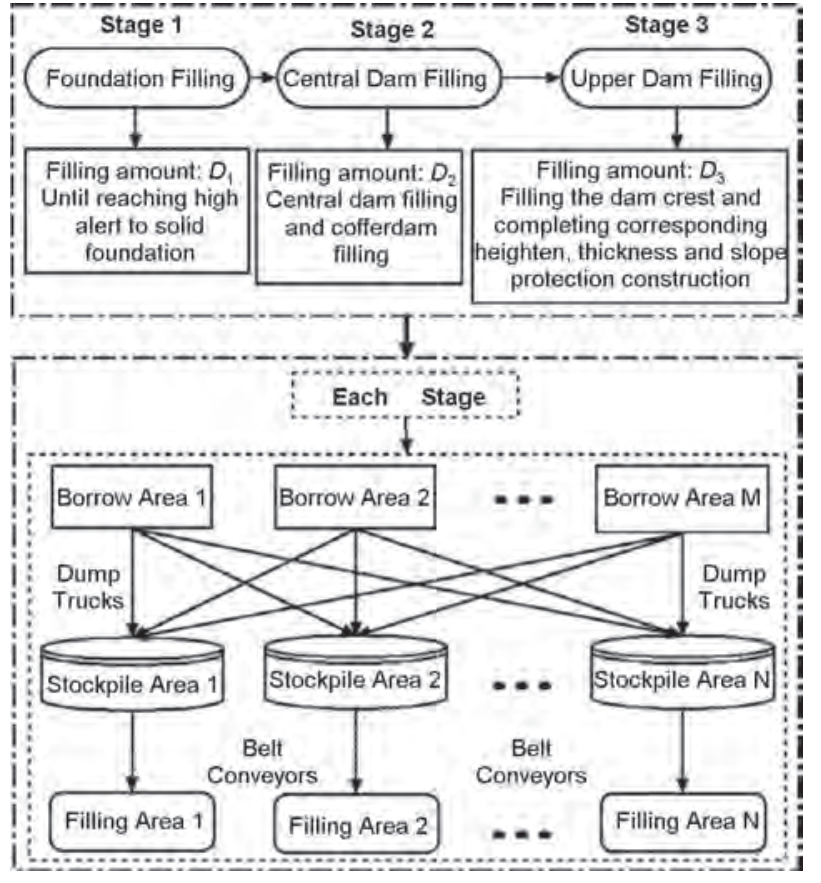

Fig. 1. Construction stages and transportation system

allocation between the borrow areas, stockpile areas and filling areas need to be appropriately planned to ensure that adequate earth-rock is available to meet the varying needs of the filling areas, and to ensure the optimization of the objectives. The TS-based DTAP proposed in this paper aims to achieve the identified objectives (i.e. cost minimization, transportation duration minimization and waste minimization) to ensure optimization and maximum benefit, efficiency and resource utilization.

\subsection{Motivation for employing fuzzy random variables in TS-based DTAP}

The need to address uncertainty in earth-rock dam construction projects is widely recognized. For example, at each stage, the earth-rock unit excavation costs may differ due to excavation difficulty uncertainty, mechanical production and construction management costs, or indirect shop labor expenses. In fact, as advancements in engineering technology have allowed for more construction operation alternatives, decision makers are faced with more imprecise information than before. Therefore, fuzzy random variables are favored by decision makers to describe the uncertainty and vague information encountered in reality. The fuzzy random environment has been successfully studied and applied in many areas, such as in inventory problems (Dutta et al. 2005; Xu, Liu 2008), portfolio problems (Ammar 2008), civil engineering (Möller, Reuter 2007), facility location-allocation problems (Xu, Liu 2011), and resource allocation problems (Xu et al. 2012c). These studies highlighted the necessity of considering the fuzzy random environment in practical problems. In practical large scale construction projects, the transportation plan is usually drawn up before any transportation 
activity commences, so the parameter data may be unknown or partially known because of the lack of statistical data, so there is a strong motivation for considering the fuzzy random environment for the TS-based DTAP.

To collect the necessary data, investigations and surveys were conducted with experienced project engineers. First, the above parameters were determined on a day to day basis, with each being an interval (i.e. $\left[l_{d}, r_{d}\right]$ ) with the highest possible value being (i.e. $m_{d}$ ). At each stage, the minimum value of all $l_{d}$ and the maximal value of all $r_{d}$ for each parameter in the survey data were determined, and these were selected as the left border (i.e. $a$ ) and the right border (i.e. $b$ ) of the fuzzy random variable, respectively. For a more accurate calculation, a new method for determining the highest possible value for each parameter at each stage is proposed, as in each stage there are many possibilities for the highest possible value, the fluctuation of which (i.e. $m_{d}$ ) is characterized by a stochastic distribution. Therefore the uncertain parameters are characterized as triangular fuzzy random variables in each stage. For example, the highest possible value (i.e. $m_{d}$ ) for the unit transportation cost can be regarded as a random variable (i.e. $\varphi(\omega))$ approximately following a normal distribution (i.e. $N\left(\mu, \eta^{2}\right)$ ), which can be estimated using the maximum likelihood method and justified by a chi-square goodness-of-fit test. Thus, the triangular fuzzy random variable for the unit transportation cost can be derived as $(a, \varphi(\omega), b)$. This modeling technique is recognized as fuzzy random optimization and from Figure 2 it can be seen why triangular fuzzy random variables are used to characterize the complex twofold uncertain environment encountered in the TS-based DTAP.

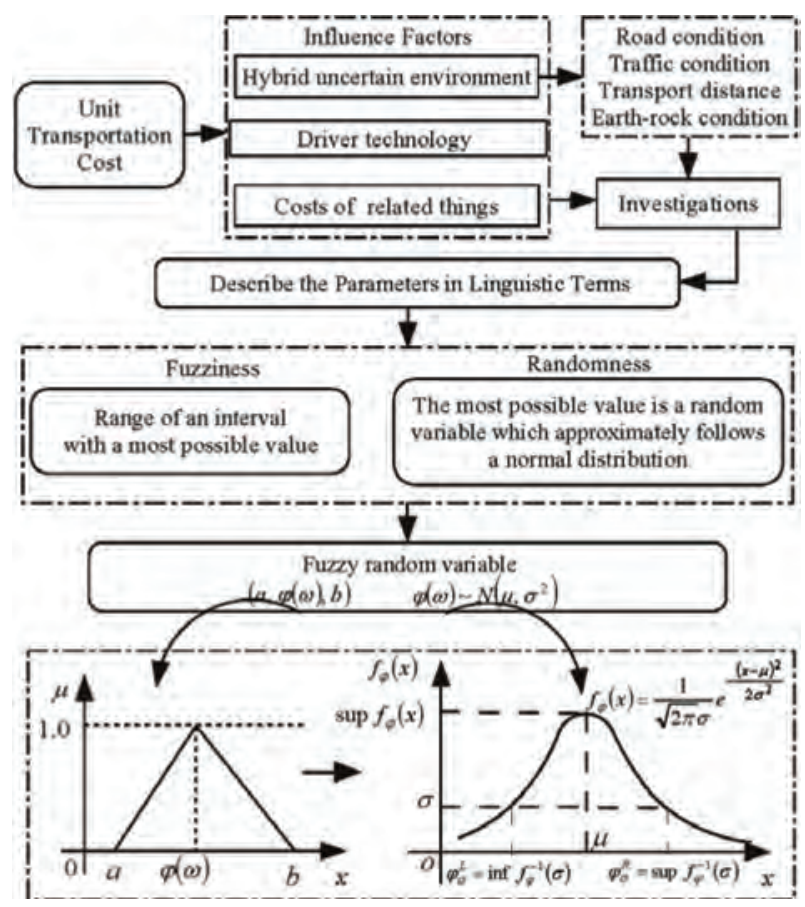

Fig. 2. Twofold uncertain environment with triangular fuzzy random variables

\section{Model formulation}

To model the objective optimization for the multistage earth-rock dam construction transportation system under a fuzzy random environment in this paper, the state transition equation, initial conditions, constraint conditions and objective functions are presented. As mentioned above, in each stage the earth-rock can be transported from any borrow area to any stockpile area, but each stockpile area is in charge of its corresponding filling area, so we can use the same set $S$ to express both the stockpile areas and filling areas. To make our model easier to understand, the following notations are introduced:

\section{Sets and subscripts}

$B$ : set of all borrow areas, $i \in B$;

$S$ : set of all stockpile areas or filling areas, $j \in S$;

$K$ : set of all stages, $k \in K$;

$A_{j}(k)$ : set of all borrow areas from which earth-rock transported to stockpile area $j$ at stage $k$.

\section{Parameters}

$\tilde{\bar{c}}_{i}(k)$ : unit excavation cost of earth-rock in borrow area $\mathrm{i}$ at stage $k, i \in B, k \in K$;

$\tilde{\bar{c}}_{j}^{1}(k)$ : unit storage cost of earth-rock in stockpile area $j$ during stage $k, j \in S, k \in K$;

$\tilde{\bar{c}}_{j}^{2}(k)$ : unit storage cost of earth-rock in stockpile area $j$ between stage $k$ and stage $k+1, j \in S, k \in K$;

$\tilde{\bar{c}}_{i j}(k)$ : unit transportation cost from borrow area $i$ to stockpile area $j$ at stage $k, i \in B, j \in S, k \in K$;

$\tilde{\bar{c}}_{i j}(k)$ : unit transportation cost from stockpile area $j$ to filling area $j$ at stage $k, j \in S, k \in K$;

$\tilde{\bar{t}}_{i j}(k)$ : unit transportation time of the first dump truck from borrow area $i$ to stockpile area $j$ at stage $k$, $i \in B, j \in S, k \in K$;

$\tilde{\bar{t}}_{j j}(k)$ : unit transportation time from stockpile area $j$ to filling area $j$ at stage $k, j \in S, k \in K$;

$\tilde{\bar{w}}_{i j}(k)$ : waste of per unit earth-rock transported from borrow area $i$ to stockpile area $j$ at stage $k, j \in S$, $k \in K$;

$\tilde{\bar{w}}_{j j}(k)$ : waste of per unit earth-rock transported from stockpile area $j$ to filling area $j$ at stage $k, j \in S$, $k \in K$; $k \in K$.

$\tilde{d}_{j}(k)$ : demand of filling area $j$ at stage $k, j \in S$,

\section{Decision variables}

$$
\delta_{i j}(k)=\left\{\begin{array}{lc}
1, & i \in A_{j}(k) \\
0, & \text { otherwise }
\end{array} ;\right.
$$

$x_{i j}(k)$ : amount of earth-rock transported from borrow area $i$ to stockpile area $j$ at stage $k, i \in A, j \in S$, $k \in K$;

$y_{i j}(k)$ : amount of earth-rock transported from stockpile area $j$ to filling area $j$ at stage $k, j \in S, k \in K$. 


\subsection{Dealing with fuzzy random variables}

Before modeling the multiple objective optimization for the multistage earth-rock dam construction transportation system, the method for dealing with the fuzzy random parameters is introduced.

While the fuzzy random variable for this problem ensures greater accuracy, its use makes the model much more difficult to solve. One strategy is to employ a transformation method to convert the fuzzy random variables into real numbers, so a deterministic model is obtained. In this paper, the hybrid crisp approach put forward in Xu et al. (2012b) is used to integrate the decision maker's optimistic-pessimistic attitudes in adopting real world practice. This method first transforms the fuzzy random parameters into $(\gamma, \sigma)$ - level trapezoidal fuzzy variables, which are subsequently defuzzified using an expected value operator with an optimistic-pessimistic index. As described before, $\tilde{\bar{c}}_{i}(k), \tilde{\bar{c}}_{j}^{1}(k), \tilde{\bar{c}}_{j}^{2}(k), \tilde{\bar{c}}_{i j}(k), \tilde{\bar{c}}_{j j}(k), \tilde{\bar{t}}_{i j}(k), \tilde{\bar{t}}_{j j}(k), \tilde{\bar{w}}_{i j}(k)$, $\tilde{\bar{w}}_{j j}(k)$ can be characterized as triangular fuzzy random numbers, generally, let $\tilde{\bar{\xi}}=\left([\xi]_{L}, \varphi(\omega),[\xi]_{R}\right)$ be any of them, where $\varphi(\omega)$ is a random variable with a probability density function $f_{\varphi}(x), \gamma$ and $\sigma$ are any given possibility level of the fuzzy variable and any given probability level of the random variable, respectively. The procedure for converting these fuzzy random variables in both the objective function and constraints into $(\gamma, \sigma)$-level trapezoidal fuzzy variables is summarized as follows:

(1) Denote the fuzzy random variable as $\tilde{\bar{\xi}}=\left([\xi]_{L}, \varphi(\omega),[\xi]_{R}\right)$. Furthermore, it is assumed that $\varphi(\omega)$ follows a normal distribution $N\left(\mu, \eta^{2}\right)$.

(2) Estimate the parameters $[\xi]_{L},[\xi]_{R}, \mu$ and $\eta^{2}$ from the collected data and professional experience using statistical methods such as the maximum likelihood estimation and the chi-square goodness-of-fit test.

(3) Obtain the decision-maker's degree of opti$\operatorname{mism} \gamma$ and $\sigma$, where $r \in\left[\frac{[\xi]_{R}-[\xi]_{L}}{[\xi]_{R}-[\xi]_{L}+\varphi_{\sigma}^{R}-\varphi_{\sigma}^{L}}, 1\right]$, $\sigma \in\left[0, \sup f_{\varphi}(x)\right]$.

(4) Let $\varphi_{\sigma}$ be the $\sigma$ - level sets (or $\sigma$-cuts)of the random variable $\varphi(\omega)$, and

$\varphi_{\sigma}=\left[\varphi_{\sigma}^{L}, \varphi_{\sigma}^{R}\right]=\left\{x \in R \mid f_{\varphi}(x) \geq \sigma\right\}$. Where,

$\varphi_{\sigma}^{L}=\inf \left\{x \in R \mid f_{\varphi}(x) \geq \sigma\right\}=\mu-\sqrt{-2 \eta^{2} \ln (\sqrt{2 \pi} \sigma \eta)}$,

$\varphi_{\sigma}^{R}=\sup \left\{x \in R \mid f_{\varphi}(x) \geq \sigma\right\}=\mu+\sqrt{-2 \eta^{2} \ln (\sqrt{2 \pi} \sigma \eta)}$.

(5) Transform the fuzzy random variable $\tilde{\bar{\xi}}=\left([\xi]_{L}, \varphi(\omega),[\xi]_{R}\right)$ into the $(\gamma, \sigma)$ - level trapezoidal fuzzy variable $\tilde{\xi}_{(\gamma, \sigma)}$ by the equation: $\tilde{\bar{\xi}} \rightarrow \tilde{\xi}_{(\gamma, \sigma)}=\left([\xi]_{L}, \underline{\xi}, \bar{\xi},[\xi]_{R}\right)$, where

$$
\underline{\xi}=[\xi]_{R}-\gamma\left([\xi]_{R}-\varphi_{\sigma}^{L}\right), \bar{\xi}=[\xi]_{L}+\gamma\left(\left[\varphi_{\sigma}^{R}-[\xi]_{L}\right) .\right.
$$

After converting the fuzzy random variables into $(\gamma, \sigma)-$ trapezoidal fuzzy variables as shown above, the $(\gamma, \sigma)$ - level trapezoidal fuzzy variables are subsequently defuzzified using an expected value operator with an optimistic-pessimistic index as follows:

$$
E^{M e}\left[\tilde{\xi}_{(\gamma, \sigma)}\right]=\frac{1-\lambda}{2}\left([\xi]_{L}+\underline{\xi}\right)+\frac{\lambda}{2}\left(\bar{\xi}+[\xi]_{R}\right),
$$

where $\lambda$ is the optimistic-pessimistic index to determine the combined attitude of a decision maker. The definition of this fuzzy measure $M e$, which is a convex combination of Pos and Nec can be found in Xu and Zhou (2011), and the basic knowledge for the measures Pos and Nec can be seen in Dubois and Prade (1978).

Based on the above method, for example, the fuzzy random variables $\tilde{\bar{c}}_{i j}(k)$ in this paper can be transformed into the $\left(\gamma_{i j}, \sigma_{i j}\right)$ - level trapezoidal fuzzy variable. It should be noted that the probability and possibility coefficients of different fuzzy random variables may be different, but in this paper, to make the model easier to solve, we set all probability levels $\sigma_{i j}, \sigma_{i j}, \sigma_{i}, \sigma_{j}, \sigma_{j}^{1}$ and $\sigma_{j}^{2}$ at the same value $\sigma$, and all possibility levels $\gamma_{i j}, \gamma_{j j}$, $\gamma_{i}, \gamma_{j}, \gamma_{j}^{1}$ and $\gamma_{j}^{2}$ at the same value $\gamma$.

\subsection{State equations}

From the decision variable notations, it can be seen that $x_{i j}(k) \delta_{i j}(k)$ expresses the amount of earth-rock transported from borrow area $i$ to stockpile area $j$ at stage $k$. It is assumed that there are $M$ borrow areas, $N$ stockpile areas

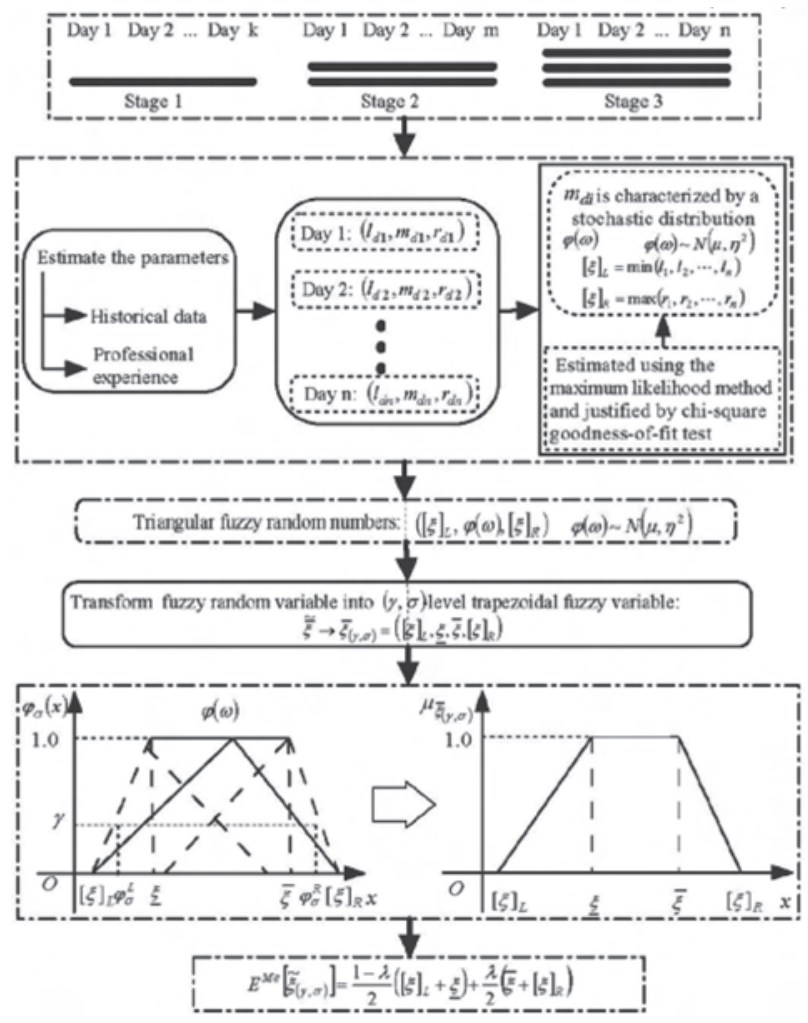

Fig. 3. Transformation method of the fuzzy random parameters 
and $N$ filling areas. Let $B_{i}(k)$ and $S_{j}(k)$ be the amount of available earth-rock in borrow area $i(i=1,2, \ldots, M)$ and stockpile area $j(j=1,2, \ldots, N)$ at the end of stage $k$, respectively. Therefore we can use Eqns (2) and (3) to describe the relationships between the state variables (i.e. $B_{i}(k)$ and $S_{j}(k)$ ) and the decision variables (i.e. $\delta_{i j}(k)$, $x_{i j}(k)$ and $\left.y_{j j}(k)\right)$, which are shown as follows:

$$
\begin{aligned}
& B_{i}(k)=B_{i}(k-1)-\sum_{j=1}^{N} x_{i j}(k) \delta_{i j}(k), \quad \forall i \in B, k \in K ;(2) \\
& S_{j}(k)=S_{j}(k-1)+\sum_{i=1}^{M} x_{i j}(k) \delta_{i j}(k) . \\
& \left(1-E^{M e}\left[\tilde{w}_{i j}(k)_{(\gamma, \sigma)}\right]\right)-y_{j j}(k), \quad \forall j \in S, k \in K . \quad(3)
\end{aligned}
$$

\subsection{Initial conditions}

It should be noted that the amount of earth-rock in every borrow area is fixed at the beginning of the construction project, and let $\alpha_{i}$ be the total amount of earth-rock in borrow area $i$. The amount of available earth-rock at stockpile area $j(j=1,2, \ldots, N)$ at the beginning of the construction project is zero. Thus the state variables $B_{i}(k)$ and $S_{i}(k)$ can be initialized as follows:

$$
\begin{gathered}
B_{i}(0)=\alpha_{i}, \quad \forall i \in B ; \\
S_{j}(0)=0, \quad \forall j \in J .
\end{gathered}
$$

\subsection{Constraint conditions}

As shown in notations, the total demand of filling area $j$ at stage $k$ (i.e. $\tilde{d}_{j}(k)$ ) is decided by the project manager according to the construction requirements at stage $k$, It is a triangular fuzzy number with $\tilde{d}_{j}(k)=\left(d_{j}^{L}(k), d_{j}^{M}(k), d_{j}^{R}(k)\right)$ wherein, $d_{j}^{L}(k)$ and $d_{j}^{R}(k)$ are the minimum and maximum of the total demand of filling area $j$ at stage $k$, respectively. From the global transportation system and construction stage view, the total amount of earth-rock in all borrow areas after considering the waste during transportation should satisfy the total maximum demand of all filling areas. The amount of earth-rock in borrow area $i$ transported to stockpile area $j$ at stage $k$ (i.e. $\left.x_{i j}(k)\right)$ should be nonnegative and not more than the total amount of available earth-rock of borrow area $i$ at the end of stage $(k-1)$ (i.e. $\left.B_{i}(k-1)\right)$. Similarly, the amount of earth-rock in stockpile area $j$ transported to its corresponding filling area should be no less than the total demand of filling area $j$ at stage $k$, and not more than the sum amount of earth-rock transported from each borrow area and the earth-rock left in stockpile area $j$ at the last stage, then according to Eqn (3), the constraint conditions are listed as follows:

$$
\begin{aligned}
& \sum_{i=1}^{M} \alpha_{i}-\sum_{k=1}^{3} \sum_{j=1}^{N}\left(\sum_{i=1}^{M} E^{M e}\left[\tilde{w}_{i j}(k)_{(\gamma, \sigma)}\right] x_{i j}(k) \delta_{i j}(k)+\right. \\
& \left.E^{M e}\left[\tilde{w}_{j j}(k)_{(\gamma, \sigma)}\right] y_{j j}(k)\right) \geq \sum_{k=1}^{3} \sum_{j=1}^{N} d_{j}^{R}(k) ;
\end{aligned}
$$

$$
\begin{gathered}
0 \leq \sum_{j=1}^{N} x_{i j}(k) \delta_{i j}(k) \leq B_{i}(k-1), \quad \forall i \in B, k \in K ; \\
y_{j j}(k) \cdot\left(1-E^{M e}\left[\tilde{w}_{j j}(k)_{(\gamma, \sigma)}\right]\right) \geq d_{j}^{L}(k), \quad \forall j \in S, k \in K \\
S_{j}(k) \geq 0, \quad \forall j \in S, k \in K .
\end{gathered}
$$

Constraint condition Eqn (9) is naturally satisfied because of the assumption that the amount of earth-rock transported to the filling area will never exceed the amount of available earth-rock at each stockpile area at any given moment.

It is assumed that each stockpile area has its own maximum capacity at the end of the stages, so let $\beta_{j}$ be the maximum capacity of stockpile area $j$. The amount of available earth-rock in stockpile $j$ at the end of stage $k$ (i.e. $S_{j}(k)$ ) should be no more than the maximum capacity of stockpile area $j$. Thus Eqn (10) is also considered a constraint condition:

$$
S_{j}(k) \leq \beta_{j}, \quad \forall j \in S, k \in K .
$$

To simplify the problem, it is assumed that the amount of earth-rock transported from borrow area $i$ to stockpile area $j$ at each stage is at least the capacity of one dump truck when $\delta_{i j}(k)=1$. Let $C$ be the heaped capacity of the dump truck. Then the following constraint condition is derived:

$$
x_{i j}(k) \geq C, \quad \forall i \in B, j \in S, k \in K .
$$

\subsection{Objective functions}

For large-scale construction projects, minimizing total cost and transportation duration are two extremely important objectives. During the transportation and construction, earth-rock work waste is inevitable, so the presented optimization model is formulated to minimize total cost and the transportation duration, while also minimizing total waste. To this end, decisions are selected by optimizing the expected values of the objective functions subject to various constraints, and the details of which are now presented.

\subsubsection{Total cost}

This objective function defines the total cost of the complete construction. Usually, total project cost varies because of many factors such as unit transportation cost, unit excavation cost, unit storage cost, and amount of earth-rock. Decision makers, therefore, aim to achieve the best option so that the total project cost (excavation cost, transportation cost and storage cost) is minimal. The details of each component are given below.

Excavation cost. In the construction project described in the problem statement, the unit excavation cost of the earth-rock in each borrow area at each stage may be different because of the uncertainty related to excavation difficulty, mechanical production and construction man- 
agement costs, and indirect shop labor expenses, so fuzzy random variables $\tilde{\overline{c_{i}}}(k)$ are used to express these characteristics. Let $C_{e}$ be the total excavation cost of the earthrock, which can be expressed as follows:

$$
C_{e}=\sum_{k=1}^{3} \sum_{i=1}^{M} \sum_{j=1}^{N} x_{i j}(k) \delta_{i j}(k) \cdot E^{M e}\left[\tilde{c}_{i}(k)_{(\gamma, \sigma)}\right] .
$$

Transportation cost. The transportation system in the TS-based DTAP discussed in this paper contains two subsystems as shown in Figure 1. The first is the transportation by dump trucks from the borrow areas to the stockpile areas, the second is the transportation using belt conveyors from the stockpile areas to their corresponding filling areas. The transportation cost for different transportation routes between different borrow areas, stockpile areas and filling areas in each stage are dissimilar and uncertain because of the differences in such elements as distance, road condition, traffic intensity, and weather. $\tilde{\bar{c}}_{i j}(k)$ and $\tilde{\bar{c}}_{j j}(k)$ are used to express the unit transportation cost of the two transportation subsystems respectively. Let $C_{t}$ be the total transportation cost, which can be expressed as follows:

$$
\begin{aligned}
C_{t}= & \sum_{k=1}^{3} \sum_{j=1}^{N} \sum_{i=1}^{M} E^{M e}\left[\tilde{c}_{i j}(k)_{(\gamma, \sigma)}\right] \cdot x_{i j}(k) \delta_{i j}(k)+ \\
& \sum_{k=1}^{3} \sum_{j=1}^{N} E^{M e}\left[\tilde{c}_{j j}(k)_{(\gamma, \sigma)}\right] \cdot y_{j j}(k) .
\end{aligned}
$$

Storage cost. The total storage cost is the storage cost at every stage and the storage cost between stages. From the notations for unit storage cost between stages and the state variables for the stockpile areas, the storage cost in stockpile area $j$ between stage $k$ and stage $(k+1)$ is $E^{M e}\left[\tilde{c}_{j}^{2}(k)_{(\gamma, \sigma)}\right] \cdot S_{j}(k)$. Let $h_{j}^{k}(t)$ express the amount of earth-rock in stockpile area $j$ at time $t$ during stage $k$. It can be seen that $h_{j}^{k}(t)$ changes over time, which leads to changes in the storage cost. Let $T_{j}(k)$ be the transportation duration at stockpile area $j$ at stage $k$, is $h_{j}^{k}(\bar{t})$ the maximum value of $h_{j}^{k}(t)$ during duration $T_{j}(k)$. Then, the storage cost in stockpile area $j$ during stage $k$ is $\int_{0}^{T_{j}(k)} \tilde{\bar{c}}_{j}^{1}(k) \cdot h_{j}^{k}(t) d t$, which is difficult to calculate. In this paper, a conversion factor is introduced to balance the difference between the maximum value $h_{j}^{k}(\bar{t})$ and the actual storage cost of stockpile area $j$ during stage $k$. Let $\tilde{\bar{c}}_{j}(k)$ express the conversion factor for stockpile area $j$ at stage $k$, which is defined by the following equation:

$$
\begin{aligned}
& \int_{0}^{T_{j}(k)} E^{M e}\left[\tilde{c}_{j}^{1}(k)_{(\gamma, \sigma)}\right] \cdot h_{j}^{k}(t) d t= \\
& E^{M e}\left[\tilde{c}_{j}^{1}(k)_{(\gamma, \sigma)}\right] \cdot h_{j}^{k}(\bar{t}), \quad \forall j \in S, k \in K,
\end{aligned}
$$

where $\tilde{\overline{c_{j}}}(k)$ is a fuzzy random number and its expected value $E^{M e}\left[\tilde{c}_{j}(k)_{(\gamma, \sigma)}\right]$ is between 0 and 1 .

Let $C_{S}$ be the total storage cost, which can be expressed as follows:

$$
\begin{aligned}
C_{s}= & \sum_{k=1}^{2} \sum_{j=1}^{N} E^{M e}\left[\tilde{c}_{j}^{2}(k)_{(\gamma, \sigma)}\right] \cdot S_{j}(k)+ \\
& \sum_{k=1}^{3} \sum_{j=1}^{N} E^{M e}\left[\tilde{c}_{j}(k)_{(\gamma, \sigma)}\right] \cdot E^{M e}\left[\tilde{c}_{j}^{1}(k)_{(\gamma, \sigma)}\right] \cdot h_{j}^{k}(\bar{t}) .
\end{aligned}
$$

Therefore, the total cost in the whole system is:

$$
f_{c}\left(x_{i j}(k), \delta_{i j}(k), y_{j j}(k)\right)=C_{e}+C_{t}+C_{s} .
$$

\subsubsection{Transportation duration}

In the transportation system, due to the distance, road condition, traffic intensity and other important influential factors, transportation time between the different borrow areas, stockpile areas and filling areas is dissimilar and uncertain. At each construction stage, earth-rock is transported by dump truck from the borrow areas to the stockpile areas, then, using belt conveyors, from the stockpile areas to the filling areas. When the first dump truck arrives at stockpile area $j$ from the different borrow areas, earthrock from stockpile area $j$ is then transported to the corresponding filling area. The dump trucks and belt conveyors are moving the earth-rock at the same time, with the belt conveyor being the last to finish. Stock out waiting time at the stockpile areas is ignored. Therefore, the transportation duration related to filling area $j$ at each stage includes the transportation time of the first dump truck arriving at stockpile area $j$ and the transportation time of all earth-rock transported from stockpile area $j$ to its corresponding filling area $j$. Let $T_{j}(k)$ and $T(k)$ be the transportation duration related to filling area $j$ at stage $k$ and the total transportation duration related to all filling areas at stage $k$ respectively, then $T_{j}(k)$ and $T(k)$ can be expressed as follows:

$$
\begin{gathered}
T_{j}(k)=\min \left\{E^{M e}\left[\tilde{t}_{i j}(k)_{(\gamma, \sigma)}\right] \mid \delta_{i j}(k)=1\right\}+ \\
E^{M e}\left[\tilde{t}_{j j}(k)_{(\gamma, \sigma)}\right] \cdot y_{j j}(k), \quad i \in A_{j}(k), j \in S, k \in K . \\
T(k)=\max _{j} T_{j}(k) .
\end{gathered}
$$

Assume that $\tilde{\bar{t}}_{i j}(k)$ is infinite when $\delta_{i j}(k)=0$, so let $\left\{\tilde{\bar{t}}_{i j}(k) \mid \delta_{i j}(k)=1\right\}$ express the transportation time of the first dump truck from borrow area $i$ to stockpile area $j$ at stage $k$. Therefore, the total transportation duration is:

$f_{d}\left(x_{i j}(k), \delta_{i j}(k), y_{j j}(k)\right)=\sum_{k=1}^{3} T(k)=\sum_{k=1}^{3} \max _{j} T_{j}(k)$.

\subsubsection{Total waste}

Earth-rock waste is inevitable because of the road condition, driving technology and other factors. Similarly, due to the difference in the road condition on different routes, and the difference on different sections of the same route, the per unit earth-rock waste is uncertain under a fuzzy random environment. At the end of each stage, the earthrock unused at filling areas is discarded. Therefore the total construction project waste is: 


$$
\begin{aligned}
f_{w}\left(x_{i j}(k), \delta_{i j}(k), y_{j j}(k)\right)= & \sum_{k=1}^{3} \sum_{j=1}^{N} \sum_{i=1}^{M} x_{i j}(k) \delta_{i j}(k)- \\
& \sum_{k=1}^{3} \sum_{j=1}^{N} E^{M e}\left[\tilde{d}_{j}(k)\right] .
\end{aligned}
$$

\subsection{Expected value model}

From the background of the earth-rock transportation system at the Pubugou Hydropower Project, appropriate planning for transportation route selection and earth-rock allocation between the borrow areas, stockpile areas and the filling areas to achieve the objectives (i.e. total cost minimization, transportation duration minimization and waste minimization) is proposed in this paper. For the state equations, constraints, and objective functions outlined above, a multistage multi-objective optimal control model is established to solve the TS-based DTAP under a fuzzy random environment. The fuzzy random coefficients are estimated using the maximum likelihood method and justified by a chi-square goodness-of-fit test. Then a hybrid crisp approach is used to transform the fuzzy random parameters into $(\gamma, \sigma)$-level trapezoidal fuzzy variables, which are subsequently defuzzified using an expected value operator with an optimistic-pessimistic index as shown in Eqn (1). Therefore, by integrating Eqns (2)-(14), the research problem TS-based DTAP can be formulated in the following Eqn (15) multi-objective expected value model:

$$
\begin{aligned}
& \sum_{k=1} \sum_{j=1} E^{M e}\left[\tilde{c}_{j}(k)_{(\gamma, \sigma)}\right] E^{M e}\left[\tilde{c}_{j}^{1}(k)_{(\gamma, \sigma)}\right] h_{j}^{k}(\bar{t}), \\
& \min f_{d}\left(x_{i j}(k), \delta_{i j}(k), y_{j j}(k)\right)=\sum_{k=1}^{3} \max _{j}\left(\min _{i}\left\{E^{M e}\left[\tilde{t}_{i j}(k)_{(\gamma, \sigma)}\right] \mid \delta_{i j}(k)=1\right\}+E^{M e}\left[\tilde{t}_{j j}(k)_{(\gamma, \sigma)}\right] y_{j j}(k),\right. \\
& \min f_{w}\left(x_{i j}(k), \delta_{i j}(k), y_{j j}(k)\right)=\sum_{k=1}^{3} \sum_{j=1}^{N} \sum_{i=1}^{M} x_{i j}(k) \delta_{i j}(k)-\sum_{k=1}^{3} \sum_{j=1}^{N} E^{M e}\left[\tilde{d}_{j}(k)\right]
\end{aligned}
$$

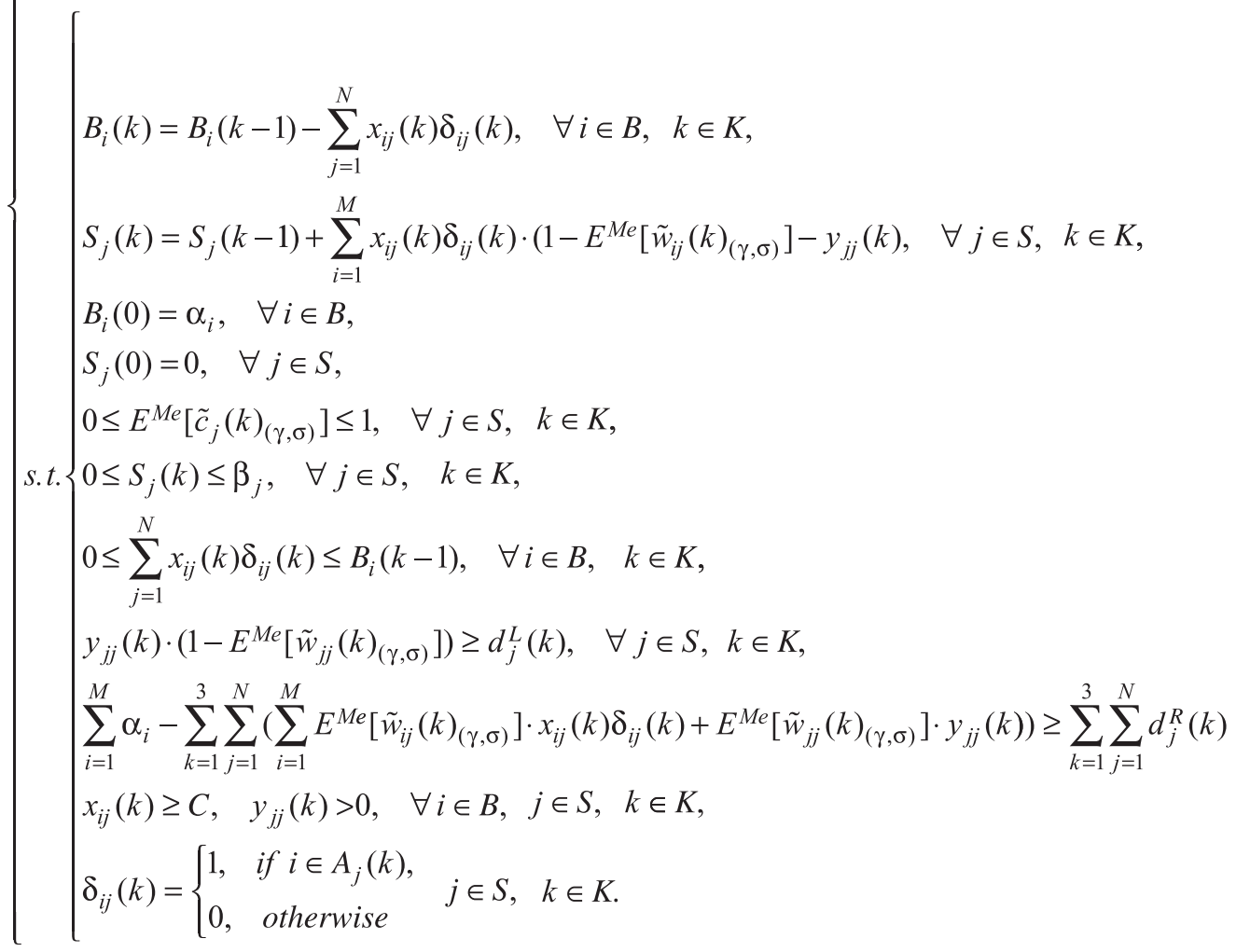

\section{Dynamic programming-based contraction particle swarm optimization}

Particle swarm optimization (PSO) is a population-based self-adaptive search stochastic optimization technique proposed by Kennedy and Eberhart (1995), which was inspired by the social behaviour of animals such as fish schooling and birds flocking to find a promising position for certain objectives in a multidimensional space (Eberhart,
Shi 2001). Similar to other population-based algorithms, such as evolutionary algorithms, PSO can solve a variety of difficult optimization problems but has shown a faster convergence rate than other evolutionary algorithms on some problems (Clerc, Kennedy 2002). There have been many variants (Anghinolfi, Paolucci 2009; Yapicioglu et al. 2007; Xu, Zeng 2011) of the PSO algorithm since its debut. Existing publications indicate that the PSO method has comparable or even superior performance when solv- 
ing many NP-hard problems and has a fast and stable convergence. As it is easy-to-implement, effective, and relatively efficient, the PSO is adopted in this study to develop a DP-based CPSO algorithm for solving our transformed optimization model.

Unlike the standard PSO, the DP-based CPSO in this paper can reduce the solution representation dimensions using the state equation from the expected value dynamic programming model (15), and each particle is represented by its position in an H-dimensional space. For the TS-based DTAP, the problem dimensions should include decision variables (i.e. $x_{i j}(k), \delta_{i j}(k)$ and $y_{i j}(k)$ and state variables (i.e. $B_{i}(k)$ and $\left.S_{j}(k)\right)$. If the decision variables are known, then the state variables can be determined based on the state equations Eqn (2) and Eqn (3). Thus, the solution representation dimensions as well as the search space can be reduced by using the state equation when developing the iterative solution algorithm for Model (15) for the TS-based DTAP. More specifically, our approach treats the state variables as hidden parameters. This in turn eliminates many redundant feasibility checks during initialization and particle updates at each iteration. The basic PSO formula is shown below:

$$
\begin{gathered}
V_{l}(\tau+1)=w(\tau) V_{l}(\tau)+c_{p} r_{p}\left(\Psi_{l}-P_{l}(\tau)\right)+ \\
c_{g} r_{g}\left(\Psi_{g}-P_{l}(\tau)\right) ; \\
P_{l}(\tau+1)=V_{l}(\tau+1)+P_{l}(\tau),
\end{gathered}
$$

where: particle index $l=1,2, \ldots, L$; is the population size; iteration index $\tau=1,2, \ldots, T$ is the iterationl limit; $V_{l}(\tau)=\left\{v_{l 1}(\tau), v_{l 2}(\tau), \ldots, v_{l h}(\tau), \ldots, v_{l H}(\tau)\right\}$ denotes the H-dimension velocity for the $l^{\text {th }}$ particle in the $\tau^{\text {th }}$ iteration, wherein, $h=1,2, \ldots, H$ is the dimension index, $H$ is the problem dimension; $P_{l}(\tau)=\left\{p_{l 1}(\tau), p_{l 2}(\tau), \ldots, p_{l H}(\tau)\right\}$ denotes the H-dimension position for the $l^{\text {th }}$ particle in the $\tau^{\text {th }}$ iteration; $\Psi_{l}=\left\{\Psi_{l 1}, \Psi_{l 2}, \ldots, \Psi_{l H}\right\}$ denotes the personal best position of the $l^{\text {th }}$ particle encountered after $\tau$ iterations; $\Psi_{g}=\left\{\Psi_{g 1}, \Psi_{g 2}, \ldots, \Psi_{g H}\right\}$ denotes the global best among all the swarm of particles achieved so far; $c_{p}$ and $c_{g}$ are the personal best and global best position acceleration constant respectively, and they determine the relative weight of the global best to the personal best; $r_{p}$ and $r_{g}$ are uniform random numbers in the interval $[0,1]$; $w(\tau)$, the inertia weight used to control the impact of the previous velocities on the current velocity that influences the trade-off between the global and the local exploration abilities during the search, and the adaptive inertia weights are set to be varying with iterations as shown in Eqn (18):

$$
w(\tau)=w(T)+\frac{\tau-T}{1-T}[w(1)-w(T)] .
$$

Eqn (16) is used to calculate the particle's new velocity according to its previous velocity and the distance of its current position from its own best experience and the group's best experience. Eqn (17) is traditionally used to update the particle moving toward a new position (Shi, Eberhart1998). The adaptive inertia weights (Shi, Eberhart1998) have a good convergent behavior in this study, which is in accordance with the results provided by Eberhart and Shi (2001).

\subsection{DP-based CPSO solution representation}

As noted earlier, based on the state equations (Eqn (2) and Eqn (3)), the state variables can be determined if the decision variables have been known, so the essential difference between the DP-based CPSO and the standard PSO is that the DP-basd CPSO takes advantage of the iterative mechanism of Model (15) by using the state equations to reduce the dimensions of the particles as well as the solution search space. In this study, the DPbased CPSO dimensions are $6 M N+3 N$ (i.e. $H=6 M N+$ $3 N$ ), which is smaller than the dimensions $6 M N+6 N+$ $3 M$ in the standard PSO. Figure 4 shows the structure of the particle and how the DP-based CPSO generates solutions for the proposed multistage, multi-objective model.

\subsection{Weight-sum procedure for dealing with the multi-objective factor}

There are many methods for dealing with the multiple objectives, such as the aggregating approach, lexicographic ordering, the sub-population approach, the Pareto-based approach, and the combined approach. As the aggregated objective in the form of a weighted-sum makes it possible to find the optimal Pareto solutions only when the solution set is convex, and the convexity of the three objectives and the constraints in the model above can be proved, meaning that the TS-based DTAP mathematical model is a convex programming and its solution set is also convex. So in this paper, the weight-sum procedure is adopted to deal with the three objectives above. Before the weight-sum procedure, the estimated maximal value is used to divide the dimensions and unify the orders of magnitude (Xu et al. 2012a) to make sure the conformity in the three different objectives is effective. The basic procedure for aggregating the three objectives is as follows:

(1) Estimate maximal value $f_{c}^{\max }, f_{d}^{\max }$ and $f_{w}^{\max }$ of $f_{c}, f_{d}$ and $f_{w}$ respectively.

(2) Calculate and standardize the $f^{\prime}{ }_{c}, f^{\prime}{ }_{d}$ and $f^{\prime}{ }_{w}$ :

$$
\left\{\begin{array}{l}
f_{c}^{\prime}=f_{c} / f_{c}^{\max } \\
f_{d}^{\prime}=f_{d} / f_{d}^{\max } \\
f_{w}^{\prime}=f_{w} / f_{w}^{\max }
\end{array} .\right.
$$

(3) The weighted-sum objective function $f$ is given by the following equation:

$$
f=\min \left(\eta_{c} f_{c}^{\prime}+\eta_{d} f_{d}^{\prime}+\eta_{w} f_{w}^{\prime}\right),
$$

where: the weights $\eta_{c}, \eta_{d}$ and $\eta_{w}$ are proposed for the total project cost, total transportation duration and the total project waste during the transportation and construction system respectively, which are provided by the decision 


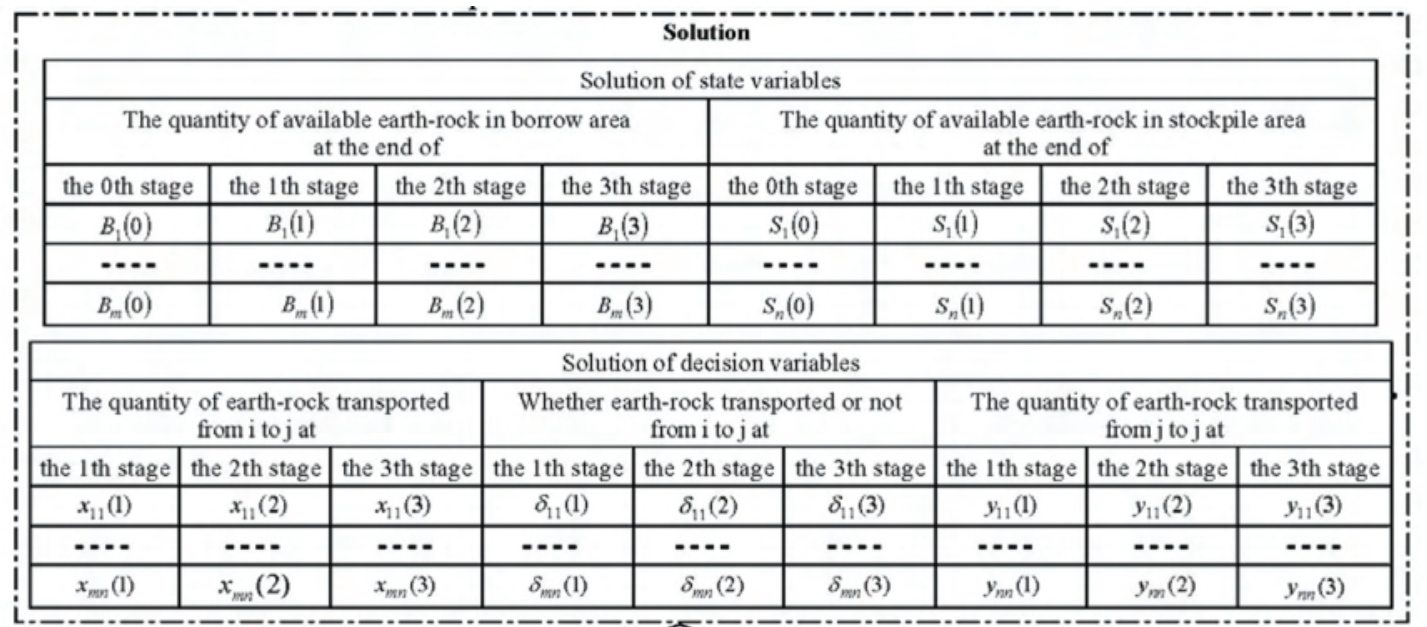
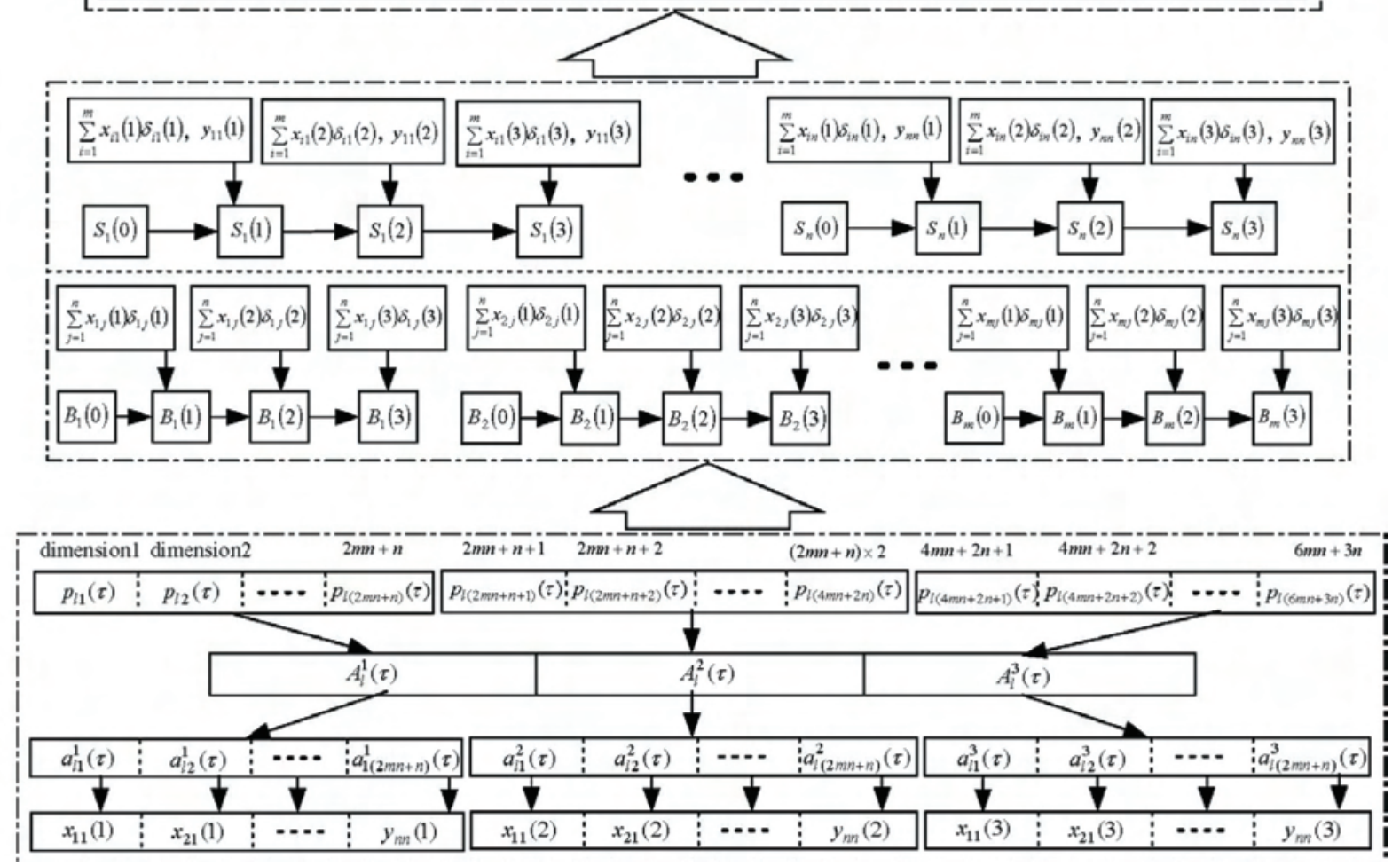

Fig. 4. Solution representation of DP-Based CPSO

makers and reflect the importance of each objective from their view. Further, these weights satisfy $\eta_{c}+\eta_{d}+\eta_{w}=1$. For a given individual, the fitness value function is expressed as follows:

$$
\operatorname{Fitness}\left(P_{l}(\tau)\right)=\eta_{c} f_{c}^{\prime}+\eta_{d} f_{d}^{\prime}+\eta_{w} f_{w}^{\prime} .
$$

\subsection{DP-based CPSO initialization}

The element in the multidimensional particle position can be initialized as follows to avoid an infeasible position: Step 1: Initialize $L$ particles as a swarm: Set iteration $\tau=1$. For $l=1,2, \ldots, L$ generate the position of particle $l$ with a random real number position $P_{l}$ in the range $\left[p^{\min }, p^{\max }\right]$. Its corresponding value is $x_{i j}(k)$, $\delta_{i j}(k)$ and $y_{j j}(k)$. From the state transition equa- tions, the state variables $B_{i}(k)$ and $S_{j}(k)$ are determined.

Step 2: Check the feasibility: All particles satisfy the constraints in the expected value Model (15), then continue. Otherwise, return to Step 1.

Step 3: Initialize the speed of each particle: For $l=1,2$, $\ldots, L$, velocity $V_{l}(1)=\left\{\vartheta_{l 1}, \vartheta_{l 2}, \cdots, \vartheta_{l H}\right\}$.

Step 4: Evaluate each of the particles: For $l=1,2, \ldots L$ compute the performance measurement of the $l^{\text {th }}$ set solution of the $h^{\text {th }}$ dimension, and set this as the fitness value of $p_{l h}(\tau)$, represented by Fitness $\left(p_{l h}(\tau)\right)$.

Step 5: Initialize the memory of each particle, the initial $\psi_{l}$ is current position $P_{l}(1)$, i.e. update personal best position $\psi_{l}=P_{l}(1)$. 


\subsection{DP-based CPSO updating and schematic procedure}

After the initialization, the updating procedure is as follows:

Step 1: WHILE the maximum number of cycles has not been reached, DO

(1) Calculate the fitness value of the objective functions $f_{c}, f_{d}$ and $f_{w}$ respectively, then calculate the fitness value Fitness $\left(P_{l}(\tau)\right)$ of the particle based on Eqn (20) and Eqn (21).

(2) Update pbest: For $l=1,2, \ldots, L$, update $\psi_{l}=P_{l}$, if Fitness $\left(P_{l}\right)<$ Fitness $\left(\psi_{l}\right)$.

(3) Update gbest: For $l=1,2, \ldots, L$, update $\psi_{g}=\psi_{l}$, if Fitness $\left(\psi_{l}\right)<$ Fitness $\left(\psi_{g}\right)$.

(4) Update the velocity and the position of each $l^{\text {th }}$ particle based on Eqn (16) Eqn (17) and Eqn (18).

(5) If $p_{l h}(\tau+1)>p^{\max }$, then:

(6)

$$
p_{l h}(\tau+1)=p^{\max }, \quad v_{l h}(\tau+1)=0 .
$$

If $p_{l h}(\tau+1)<p^{\max }$, then:

$$
p_{l h}(\tau+1)=p^{\min }, \quad v_{l h}(\tau+1)=0,
$$

where $p^{\max }$ and $p^{\min }$ are the maximum and minimum position value, respectively.

(7) Check the feasibility and make adjustments: After the above updating, the decision variables (i.e. $x_{i j}(k)$, $\delta_{i j}(k)$ and $\left.\mathrm{y}_{j j}(k)\right)$ are determined in accordance with the properties and characteristics of the constraint conditions. If some constraint conditions are not satisfied, the adjustment is made as follows:

1) Through the above updating, if $\delta_{i j}(k)$ is not a $0-1$ variable, it will be adjusted as follows:

$$
\hat{\delta}_{i j}(k)=\left\{\begin{array}{ll}
1, & \delta_{i j}(k) \geq 0.8 \\
0, & \delta_{i j}(k)<0.8
\end{array} .\right.
$$

Then, assume that the $0-1$ variable $\hat{\delta}_{i j}(k)$ does not change, no matter what constraint conditions are not satisfied;

2) Adjust $y_{j j}(k)$ according to the unsatisfied constraint conditions which do not contain $x_{i j}(k)$ such as Eqn (8). As the $\hat{\delta}_{i j}(k)$ has been decided, if $y_{i j}(k)$ does not satisfy Eqn (8), an adjustment will be made as shown in step 1.(5);

3) After the adjustment above, $\hat{\delta}_{i j}(k)$ and $\hat{y}_{j j}(k)$ are decided, then $x_{i j}(k)$ is adjusted according to the unsatisfied constraint conditions. Let $H$ and $H_{1}$ be the number of $\delta_{i j}(k)$ and $\delta_{i j}(k)=1$ in one unsatisfied constraint condition respectively. If the unsatisfied constraint condition in the remainder of the constraint conditions is in the form of $\sum_{h=1}^{H}(x \delta)_{h} \leq A$. then every $\left\{x_{i j}(k) \mid \delta_{i j}(k)=1\right\}$. should reduce $A / H_{1}$. In the model above, the remainder of the constraint conditions are shown in Eqn (6), Eqn (7) and Eqn (10) (Converted into
$S_{j}(k-1)+\sum_{i=1}^{M} x_{i j}(k) \delta_{i j}(k) \cdot\left(1-E^{M e}\left[\tilde{w}_{i j}(k)_{(\gamma, \sigma)}\right]\right)-$

$y_{j j}(k) \leq \beta_{j}$ based on Eqn (3)) and they all have the same form $\sum_{h=1}^{H}(x \delta)_{h} \leq A$. Further, if there is more than one constraint condition not satisfied, then in the whole model every $\left\{x_{i j}(k) \mid \delta_{i j}(k)=1\right\}$. should reduce $\max _{r}\left\{\left(A / H_{1}\right)_{r}\right\}$, where $r$ is the number of unsatisfied constraint conditions in the rest of the constraint conditions, so the adjustment to $x_{i j}(k)$ is $\hat{x}_{i j}(k)=x_{i j}(k)-\max _{r}\left\{\left(A / H_{1}\right)_{r}\right\} ;$

4) After the adjustment above, if there are still no feasible solutions $\hat{y}_{j j}(k)$ and $\hat{x}_{i j}(k)$, then adjust $\hat{\delta}_{i j}(k)$ again, find $\left\{E^{M e}\left[\tilde{t}_{i j}(k)_{(\gamma, \sigma)}\right] \mid \delta_{i j}(k)=0\right\}$, where $\left(E^{M e}\left[\tilde{t}_{i j}(k)_{(\gamma, \sigma)}\right] \mid \delta_{i j}(k)=0\right)$ $\leq\left(E^{M e}\left[\tilde{t}_{i j}(k)_{(\gamma, \sigma)}\right] \mid \delta_{i j}(k)>0\right)$, and choose one or more $\delta_{i j}(k)$ properly and let $\delta_{i j}(k)=1$. Continuously adjust using the above methods until all equations are satisfied;

5) After the adjustment for $x_{i j}(k), \delta_{i j}(k)$ and $y_{j j}(k)$, make a generation into the state equation and determine the state variables.

Step 2: END WHILE.

Step 3: If the stopping criterion is met, i.e. $\tau=T$, go to Step 4. Otherwise, $\tau=\tau+1$ and return to Step 1

Step 4: Decode the global best position $\Psi_{g}$ as the solution set.

Figure 5 shows the schematic procedure for the DPbased CPSO to generate solutions for the proposed multistage and multi-objective model.

\section{Practical application to a construction project}

\subsection{Project description}

In this section, an earth-rock transportation system in the Pubugou hydropower earth-rock dam construction project is taken as an example to demonstrate the optimization method. The Pubugou Hydropower project has 3300, MW installed capacity and 14.58, GWh annual output. It operates across Hanyuan County and Ganluo County, which are located in the middle reaches of the Dadu River in Sichuan province, China. The Pubugou Hydropower dam is an earth-rock dam with a gravel soil core, and is 186 meters high with a dam crest elevation of 856 meters. The earth-rock dam consists of a core wall anti-seepage material area, an upstream and downstream inverse filter material area, a filtration material area, two earth-rock filling areas, and a slope protection block stone area. The top and bottom elevations of the core wall are 856 meters and 854 meters respectively, with the top and bot- 


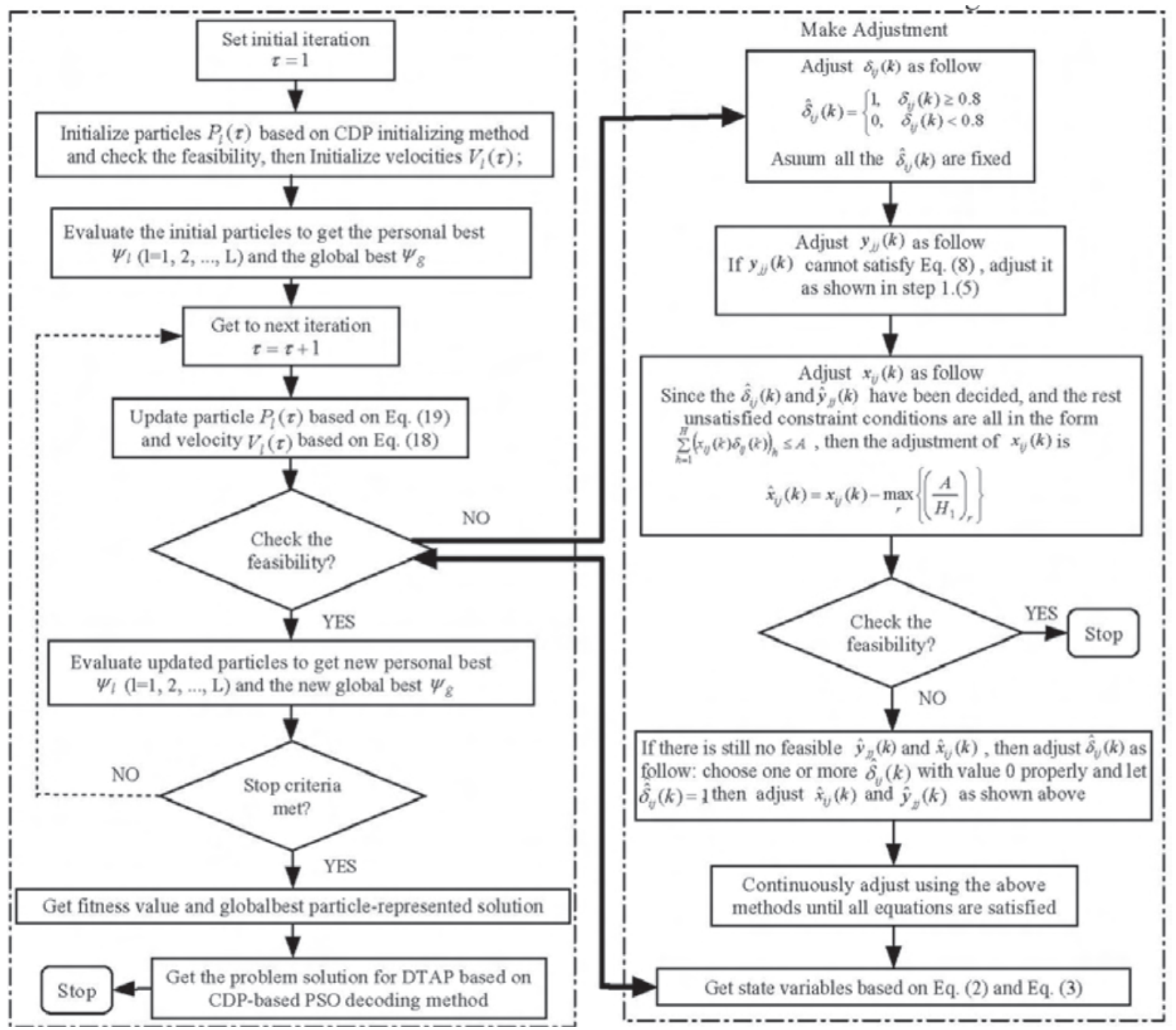

Fig. 5. Procedure of the DP-based CPSO framework

tom width being 4 meters and 96 meters, and the slope of the upstream and downstream both being 1:0.25. The total amount of dam filling is $2364.25 \times 10^{4}$ cubic meters.

\subsection{Data collection}

All detailed engineering data for the Pubugou Hydropower Project (PHP) were obtained from the Dadu River Basin Hydropower Project Construction Company. In a large hydropower construction project, earth-rock is usually the primary activity, so earth-rock transportation occurs every day in the excavation projects, the borrow areas, the filling areas and the stockpile areas as the material is turned over and thus needs to be replaced frequently. The transportation network is shown in Figure 7 and includes an external and an internal road network. The internal road has two belt conveyors, with one located on the left bank and the other on the right, and the transportation routes are fixed. The external road network has four borrow areas and two stockpile areas, and it is possible that the earth-rock could be transported from any of the borrow areas to any of the stockpile areas.
Three types of transportation equipment (dump truck and two types of belt conveyor) are used in the construction project, which transport the earth-rock along different routes between different borrow areas and stockpile areas, and then from the stockpile area to its corresponding filling area, with the destination nodes having the practical demand of timeliness. All necessary data for each carrier type were calculated as shown in Table 1. Table 2 shows the detail for each borrow area and stockpile area. To apply the proposed methods conveniently, adjacent roads of the same type were combined and road shapes were ignored.

To collect the unit cost, time and waste data, investigations and surveys were conducted to obtain historical data from both the financial department and experienced Dadu River Basin Hydropower Project Construction Company construction team engineers. Since the above parameters change over time, the data were classified based on different periods. First, they were determined on a day to day basis, and each of them was prescribed an interval (i.e. $\left[l_{d}, m_{d}, r_{d}\right]$ ). For each stage, the mini- 


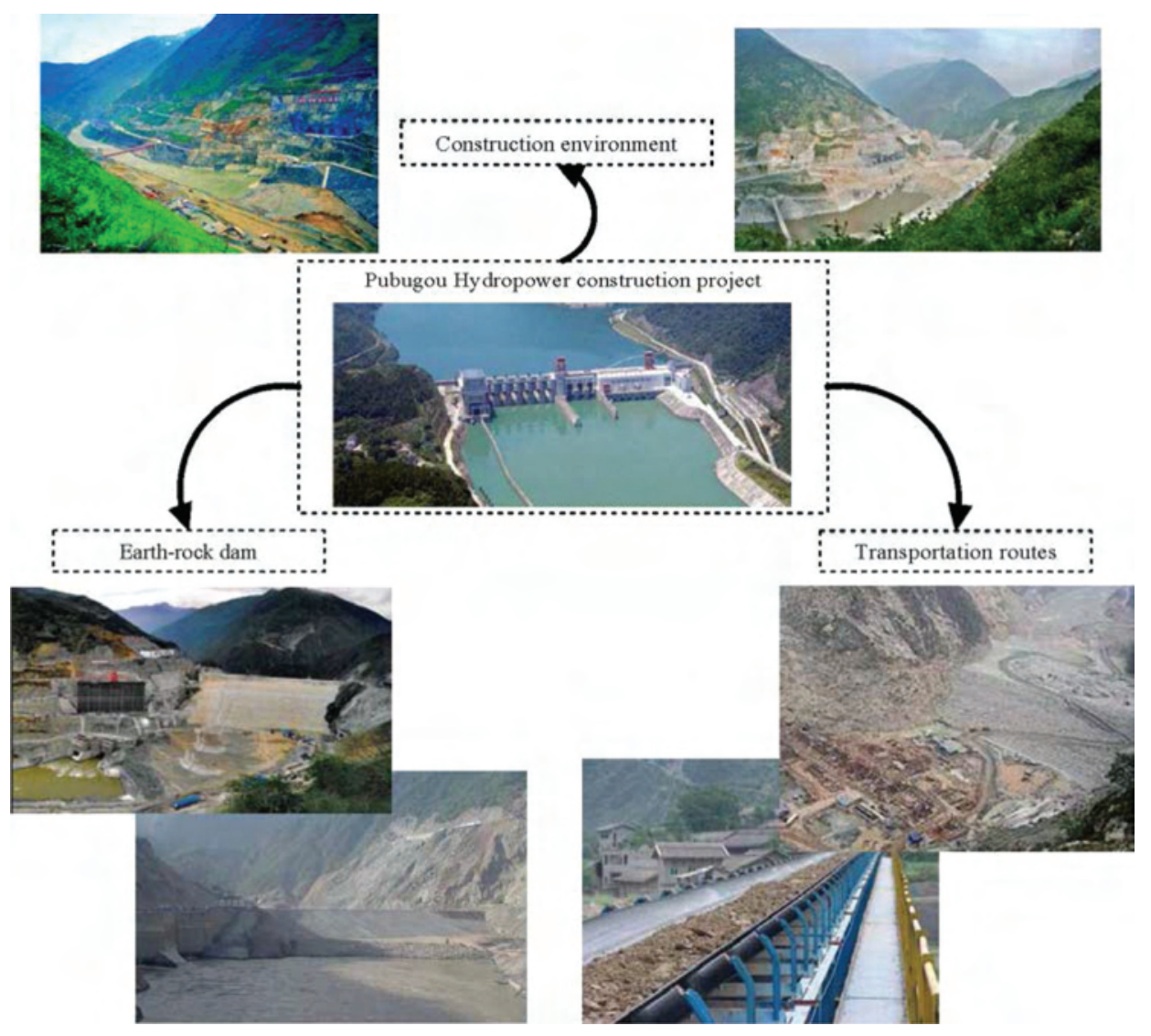

Fig. 6. Pubugou hydropower construction project

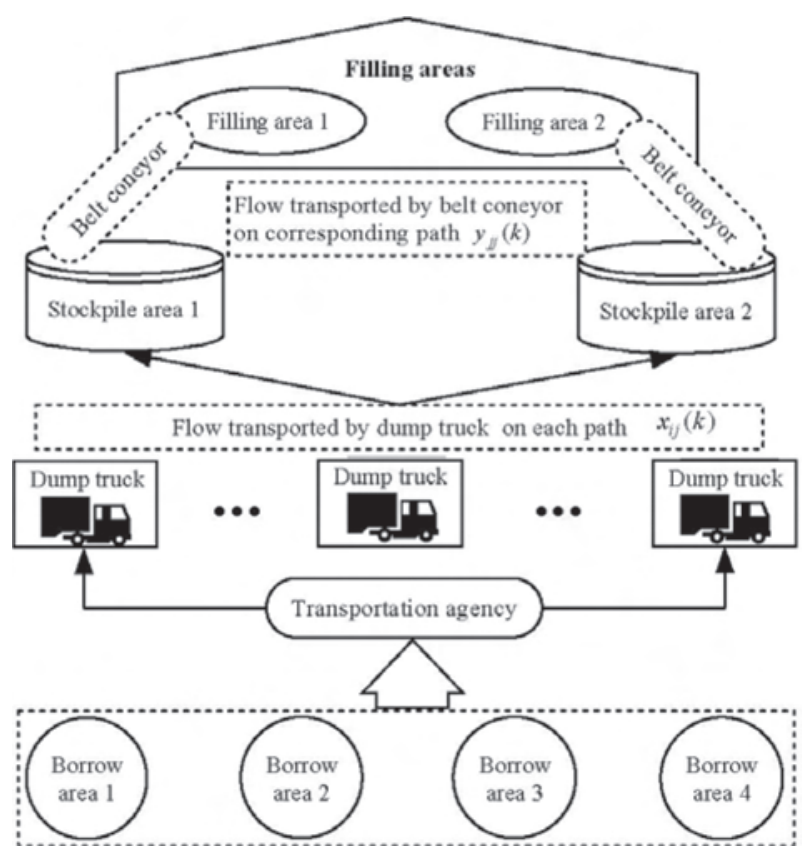

Fig. 7. Location and detailed information of borrow areas, stockpile areas and fillingareas in PHP

mum value of all $l_{d}$ and the maximal value of all $r_{d}$ for each parameter in the survey data were determined, and they were selected as the left border (i.e. $a$ ) and the right border (i.e. $b$ ) of the fuzzy random variable, respectively.
Table 1. Information of carriers in PHP

\begin{tabular}{lcl}
\hline Carriers & Kind index & \multicolumn{1}{c}{ Type } \\
\hline Dump truck & 1 & Terex TA 28(24 m³ $35 \mathrm{t})$ \\
\hline Belt conveyer & 2 & BC $(1000 \mathrm{~mm} ; 4 \mathrm{~km})$ \\
\hline Belt conveyer & 3 & $\mathrm{BC}(1200 \mathrm{~mm} ; 4.5 \mathrm{~km})$ \\
\hline
\end{tabular}

Table 2. Information of borrow areas and stockpile areas in PHP

\begin{tabular}{lcc}
\hline Areas & Area index & Maximum capacity \\
\hline Borrow area & 1 & $732.4 \times 10^{4} \mathrm{~m}^{3}$ \\
\hline Borrow area & 2 & $581.7 \times 10^{4} \mathrm{~m}^{3}$ \\
\hline Borrow area & 3 & $528.3 \times 10^{4} \mathrm{~m}^{3}$ \\
\hline Borrow area & 4 & $790.2 \times 10^{4} \mathrm{~m}^{3}$ \\
\hline Stockpile area & 1 & $15 \times 10^{4} \mathrm{~m}^{3}$ \\
\hline Stockpile area & 2 & $15 \times 10^{4} \mathrm{~m}^{3}$ \\
\hline
\end{tabular}

For a more accurate calculation, a new method for determining the highest possible value for each parameter at each stage is proposed, as in each stage there are many possibilities for the highest possible value, the fluctuation of which (i.e. $m_{d}$ ) is characterized by a stochastic distribution, which is estimated using maximum likelihood method and justified by a chi-square goodness-of-fit test. Therefore the uncertain parameters are characterized as triangular fuzzy random variables in each stage. 


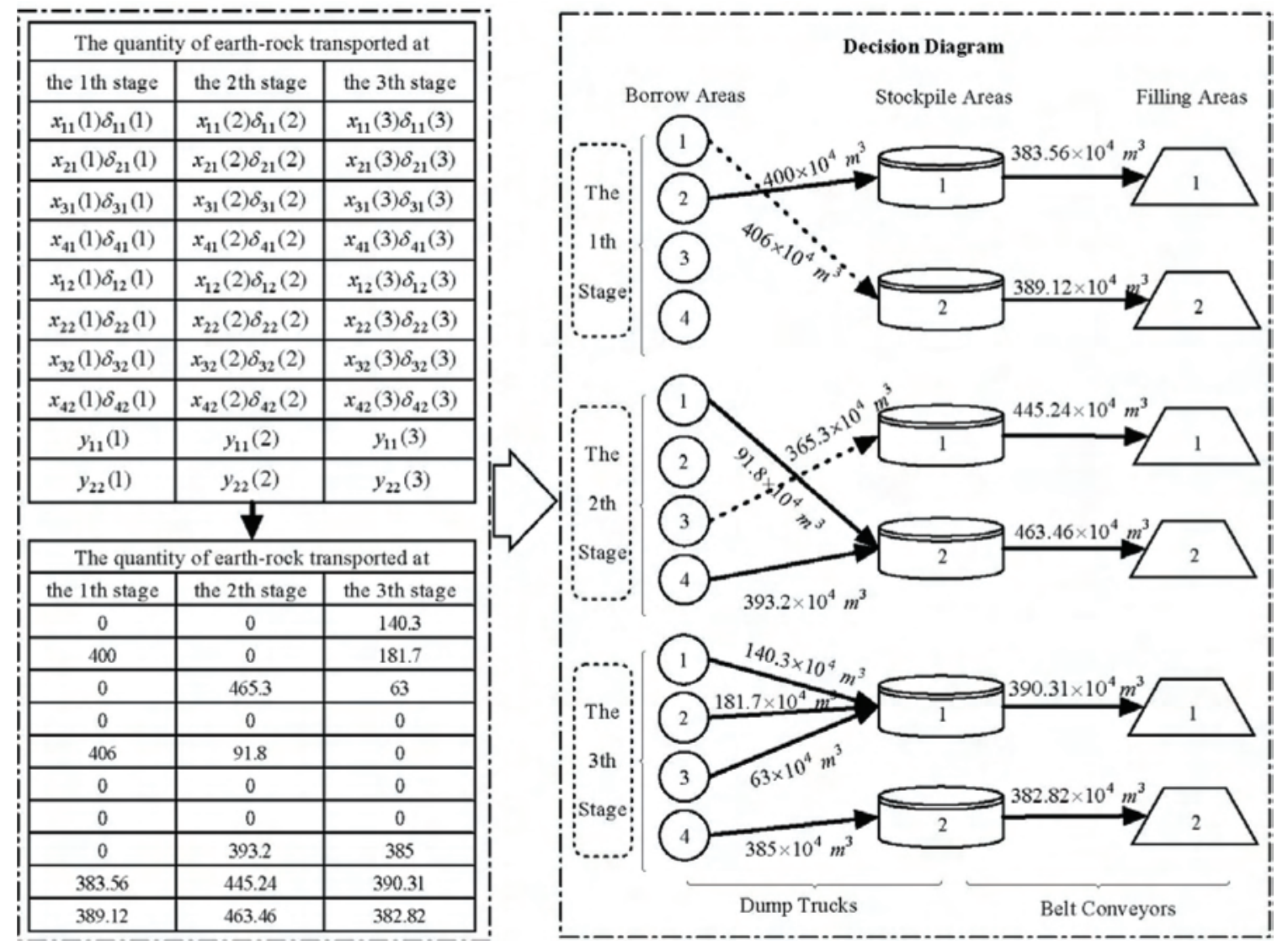

Fig. 8. Results of DP-based CPSO Algorithm for transportation allocation decision in Pubugou hydropower project

For each link in Figure 7 for the transportation network, there are free flow $L-R$ fuzzy random travel time $\tilde{\overline{t_{i j}}}(k)$ and $\tilde{\bar{t}}_{j j}(k)$, unit cost $\tilde{\bar{c}}_{i j}(k)$ and $\tilde{\bar{c}}_{j j}(k)$, and unit waste $\tilde{\bar{w}}_{i j}(k)$ and $\tilde{\bar{w}}_{j j}(k)$ during the transportation. The corresponding data are in Table 3, Table 4 and Table 5. Similarly, the unit excavation cost and unit storage cost of the earth-rock at each stage are also $L-R$ fuzzy random parameters, the data for which are stated in Table 6 . Let $\tilde{\xi}=\left([\xi]_{L}, \varphi(\omega),[\xi]_{R}\right)$ be any of above parameters, where $\varphi(\omega)$ is a random variable approximately following a Normal distribution $N\left(\mu, \eta^{2}\right)$. A hybrid crisp approach is used in this paper to transform the fuzzy random parameters into $(\gamma, \sigma)$ - level trapezoidal fuzzy variables, which are subsequently defuzzified using an expected value operator with an optimistic-pessimistic index, from which the final data is determined. Here, $\gamma$ and $\sigma$ are any given possibility level of fuzzy variable and any given probability level of random variable, respectively.

\subsection{Parameters selection for DP-based CPSO}

From the results of preliminary experiments which were carried out to observe the behavior of the algorithm at different parameter settings, and through a comparison of several sets of parameters, including population size, iter- ation number, acceleration constant, initial velocity, and inertia weight, the most suitable parameters were identified. Table 7 summarizes some of the parameter values selected for the DP-based CPSO in the computational experiments. Note that the population size determines the evaluation runs which impact the optimization cost, and various learning factors $c_{p}$ and $c_{g}$ may lead to small differences in the PSO's performance. The inertia weight $w(\tau)$ is set to be varying with iterations as follows:

$$
w(\tau)=w(T)+\frac{\tau-T}{1-T}[w(1)-w(T)],
$$

where iteration index $\tau=1,2, \ldots, T, w(1)=0.9$ and $w(T)=$ 0.1 are found to be the most suitable to control the impact of the previous velocities on the current one and influence the trade-off between the global and local experiences.

\subsection{Computational results and sensitivity analysis}

Compared with the actual data (total cost, transportation duration and waste) from the Pubugou Hydropower Project, the practicality and efficiency of the optimization model under a fuzzy random environment presented in this paper are verified through the implementation of the DP-based CPSO algorithm to solve the flow assignment between the transportation routes described previously. After running the proposed DP-based CPSO using 
Table 3. Data information of unit transportation cost in each route at each stage

\begin{tabular}{|c|c|c|c|c|}
\hline \multirow{2}{*}{\multicolumn{2}{|c|}{ Model parameters }} & \multicolumn{3}{|c|}{ Stage index } \\
\hline & & $k=1$ & $k=2$ & $k=3$ \\
\hline \multirow{16}{*}{$\begin{array}{c}\tilde{\bar{c}}_{i j}(k) \\
\text { (CNY/unit) }\end{array}$} & \multirow{2}{*}{$\tilde{\bar{c}}_{11}(k)$} & $(4.82, \varphi(\omega), 5.56)$ & $(5.26, \varphi(\omega), 6.44)$ & $(5.28, \varphi(\omega), 6.18)$ \\
\hline & & $\varphi(\omega) \sim N(5.20,3.1)$ & $\varphi(\omega) \sim N(6.00,4.2)$ & $\varphi(\omega) \sim N(5.82,3.8)$ \\
\hline & \multirow{2}{*}{$\tilde{\bar{c}}_{21}(k)$} & $(3.02, \varphi(\omega), 3.57)$ & $(3.23, \varphi(\omega), 4.09)$ & $(3.18, \varphi(\omega), 4.25)$ \\
\hline & & $\varphi(\omega) \sim N(3.25,2.1)$ & $\varphi(\omega) \sim N(3.66,2.2)$ & $\varphi(\omega) \sim N(3.72,1.8)$ \\
\hline & \multirow{2}{*}{$\tilde{\bar{c}}_{31}(k)$} & $(3.89, \varphi(\omega), 4.67)$ & $(3.97, \varphi(\omega), 4.55)$ & $(4.16, \varphi(\omega), 4.83)$ \\
\hline & & $\varphi(\omega) \sim N(4.23,1.7)$ & $\varphi(\omega) \sim N(4.34,2.1)$ & $\varphi(\omega) \sim N(4.59,2.4)$ \\
\hline & \multirow{2}{*}{$\tilde{\bar{c}}_{41}(k)$} & $(5.78, \varphi(\omega), 6.47)$ & $(6.04, \varphi(\omega), 6.58)$ & $(6.06, \varphi(\omega), 6.75)$ \\
\hline & & $\varphi(\omega) \sim N(6.12,3.8)$ & $\varphi(\omega) \sim N(6.44,4.2)$ & $\varphi(\omega) \sim N(6.40,4.0)$ \\
\hline & \multirow{2}{*}{$\tilde{\bar{c}}_{12}(k)$} & $(5.22, \varphi(\omega), 5.91)$ & $(5.04, \varphi(\omega), 5.65)$ & $(5.30, \varphi(\omega), 6.09)$ \\
\hline & & $\varphi(\omega) \sim N(5.57,2.8)$ & $\varphi(\omega) \sim N(5.41,3.0)$ & $\varphi(\omega) \sim N(5.77,4.2)$ \\
\hline & \multirow{2}{*}{$\tilde{\bar{c}}_{22}(k)$} & $(5.97, \varphi(\omega), 6.66)$ & $(6.18, \varphi(\omega), 6.47)$ & $(6.06, \varphi(\omega), 6.75)$ \\
\hline & & $\varphi(\omega) \sim N(6.21,4.1)$ & $\varphi(\omega) \sim N(6.33,4.2)$ & $\varphi(\omega) \sim N(6.50,3.8)$ \\
\hline & \multirow{2}{*}{$\tilde{\bar{c}}_{32}(k)$} & $(5.36, \varphi(\omega), 5.94)$ & $(5.27, \varphi(\omega), 5.65)$ & $(5.32, \varphi(\omega), 6.01)$ \\
\hline & & $\varphi(\omega) \sim N(5.60,3.1)$ & $\varphi(\omega) \sim N(5.41,3.0)$ & $\varphi(\omega) \sim N(5.77,4.2)$ \\
\hline & \multirow{2}{*}{$\tilde{\bar{c}}_{42}(k)$} & $(3.38, \varphi(\omega), 3.87)$ & $(3.56, \varphi(\omega), 4.09)$ & $(3.82, \varphi(\omega), 4.35)$ \\
\hline & & $\varphi(\omega) \sim N(3.63 .2 .1)$ & $\varphi(\omega) \sim N(3.84,2.2)$ & $\varphi(\omega) \sim N(4.00,2.0)$ \\
\hline \multirow{4}{*}{$\begin{array}{c}\tilde{\bar{c}}_{i j}(k) \\
\text { (CNY/unit) }\end{array}$} & \multirow{2}{*}{$\tilde{\bar{c}}_{11}(k)$} & $(3.08, \varphi(\omega), 3.40)$ & $(3.30, \varphi(\omega), 3.84)$ & $(3.36, \varphi(\omega), 3.78)$ \\
\hline & & $\varphi(\omega) \sim N(3.34,2.2)$ & $\varphi(\omega) \sim N(3.47,2.1)$ & $\varphi(\omega) \sim N(3.62,2.6)$ \\
\hline & \multirow{2}{*}{$\tilde{\bar{c}}_{22}(k)$} & $(3.07, \varphi(\omega), 3.46)$ & $(3.08, \varphi(\omega), 3.50)$ & $(3.16, \varphi(\omega), 3.65)$ \\
\hline & & $\varphi(\omega) \sim N(3.21,2.0)$ & $\varphi(\omega) \sim N(3.27,2.2)$ & $\varphi(\omega) \sim N(3.40,1.8)$ \\
\hline
\end{tabular}

MATLAB 7.0, the computational results were obtained based on an optimistic-pessimistic index $\lambda=0.5$, weights $\eta_{c}=0.5, \eta_{d}=0.3$ and $\eta_{w}=0.2$, probability $\sigma=0.2$ and possibility $\gamma=0.8$, where the maximal values of $f_{c}, f_{d}$ and $f_{w}$ were estimated and $f_{c}^{\max }=628.47 \times 10^{6}, f_{d}^{\max }=285.32 \times 10^{1}$ and $f_{w}^{\max }=196.61 \times 10^{4}$. The results and performance of the algorithm, as compared to the actual data from the project, are listed in Table 8 , and Figure 8 shows the results of the DP-based CPSO Algorithm for the transportation allocation decision at the Pubugou Hydropower Project.

In this paper, since there are some undetermined parameters such as the optimistic-pessimistic index $\lambda$, weights $\eta_{c}, \eta_{d}$ and $\eta_{w}$, probability level $\sigma$ and possibility level $\gamma$, the data for which were provided by deci- sion makers, therefore, based on the proposed model, further research should be done to analyze its sensitivity and its advantages compared with other models and algorithms.

\subsubsection{Sensitivity analysis for the optimistic-pessimistic index}

The results above were obtained based on $\lambda=0.5$, $\eta_{c}=0.5, \eta_{d}=0.3$ and $\eta_{w}=0.2, \sigma=0.2$ and $\gamma=0.8$. As discussed before, as some parameters in this paper are fuzzy random variables, a hybrid crisp approach is used in this paper to transform the fuzzy random parameters into $(\gamma, \sigma)$ - level trapezoidal fuzzy variables, which are subsequently defuzzified using an expected value operator with an optimistic-pessimistic index $\lambda$. 
Table 4. Data information of transportation time in each route at each stage

\begin{tabular}{|c|c|c|c|c|}
\hline \multirow{2}{*}{\multicolumn{2}{|c|}{ Model parameters }} & \multicolumn{3}{|c|}{ Stage index } \\
\hline & & $k=1$ & $k=2$ & $k=3$ \\
\hline \multirow{16}{*}{$\begin{array}{l}\tilde{\overline{t_{i j}}}(k) \\
\text { (hour) }\end{array}$} & \multirow{2}{*}{$\tilde{\bar{t}}_{11}(k)$} & $(0.28, \varphi(\omega), 0.40)$ & $(0.30, \varphi(\omega), 0.44)$ & $(0.26, \varphi(\omega), 0.38)$ \\
\hline & & $\varphi(\omega) \sim N(0.34,0.21)$ & $\varphi(\omega) \sim N(0.37,0.22)$ & $\varphi(\omega) \sim N(0.32,0.18)$ \\
\hline & \multirow{2}{*}{$\tilde{\bar{t}}_{21}(k)$} & $(0.20, \varphi(\omega), 0.27)$ & $(0.23, \varphi(\omega), 0.29)$ & $(0.18, \varphi(\omega), 0.25)$ \\
\hline & & $\varphi(\omega) \sim N(0.25,0.1)$ & $\varphi(\omega) \sim N(0.26,0.15)$ & $\varphi(\omega) \sim N(0.22,0.18)$ \\
\hline & \multirow{2}{*}{$\tilde{\bar{t}}_{31}(k)$} & $(0.19, \varphi(\omega), 0.27)$ & $(0.17, \varphi(\omega), 0.25)$ & $(0.16, \varphi(\omega), 0.23)$ \\
\hline & & $\varphi(\omega) \sim N(0.23,0.12)$ & $\varphi(\omega) \sim N(0.21,0.1)$ & $\varphi(\omega) \sim N(0.22,0.18)$ \\
\hline & \multirow{2}{*}{$\tilde{\bar{t}}_{41}(k)$} & $(0.38, \varphi(\omega), 0.47)$ & $(0.40, \varphi(\omega), 0.48)$ & $(0.36, \varphi(\omega), 0.45)$ \\
\hline & & $\varphi(\omega) \sim N(0.42,0.21)$ & $\varphi(\omega) \sim N(0.44,0.32)$ & $\varphi(\omega) \sim N(0.40,0.28)$ \\
\hline & \multirow{2}{*}{$\tilde{\bar{t}}_{12}(k)$} & $(0.22, \varphi(\omega), 0.31)$ & $(0.24, \varphi(\omega), 0.35)$ & $(0.20, \varphi(\omega), 0.29)$ \\
\hline & & $\varphi(\omega) \sim N(0.27,0.11)$ & $\varphi(\omega) \sim N(0.30,0.22)$ & $\varphi(\omega) \sim N(0.24,0.12)$ \\
\hline & \multirow{2}{*}{$\tilde{\bar{t}}_{22}(k)$} & $(0.17, \varphi(\omega), 0.26)$ & $(0.18, \varphi(\omega), 0.27)$ & $(0.18, \varphi(\omega), 0.25)$ \\
\hline & & $\varphi(\omega) \sim N(0.21,0.08)$ & $\varphi(\omega) \sim N(0.23,0.12)$ & $\varphi(\omega) \sim N(0.20,0.12)$ \\
\hline & \multirow{2}{*}{$\tilde{\bar{t}}_{32}(k)$} & $(0.36, \varphi(\omega), 0.44)$ & $(0.37, \varphi(\omega), 0.45)$ & $(0.32, \varphi(\omega), 0.41)$ \\
\hline & & $\varphi(\omega) \sim N(0.40,0.23)$ & $\varphi(\omega) \sim N(0.41,0.22)$ & $\varphi(\omega) \sim N(0.37,0.24)$ \\
\hline & \multirow{2}{*}{$\tilde{\bar{t}}_{42}(k)$} & $(0.28, \varphi(\omega), 0.37)$ & $(0.26, \varphi(\omega), 0.39)$ & $(0.22, \varphi(\omega), 0.35)$ \\
\hline & & $\varphi(\omega) \sim N(0.33,0.20)$ & $\varphi(\omega) \sim N(0.34,0.22)$ & $\varphi(\omega) \sim N(0.29,0.16)$ \\
\hline \multirow{4}{*}{$\begin{array}{c}\tilde{\bar{t}}_{j j}(k) \\
\text { (hour/ } 10^{4} \mathrm{~m}^{3} \text { ) }\end{array}$} & \multirow{2}{*}{$\tilde{\bar{t}}_{11}(k)$} & $(1.7, \varphi(\omega), 2.2)$ & $(1.9, \varphi(\omega), 2.3)$ & $(1.7, \varphi(\omega), 2.2)$ \\
\hline & & $\varphi(\omega) \sim N(2.0,1.4)$ & $\varphi(\omega) \sim N(2.1,1.2)$ & $\varphi(\omega) \sim N(1.9,1.0)$ \\
\hline & \multirow{2}{*}{$\tilde{\bar{t}}_{22}(k)$} & $(1.6, \varphi(\omega), 2.1)$ & $(1.8, \varphi(\omega), 2.1)$ & $(1.3, \varphi(\omega), 2.0)$ \\
\hline & & $\varphi(\omega) \sim N(1.8,1.1)$ & $\varphi(\omega) \sim N(2.0,1.2)$ & $\varphi(\omega) \sim N(1.7,1.1)$ \\
\hline
\end{tabular}

To gain further insight into the optimistic-pessimistic index selection principle in Eqn (1), a sensitivity analysis was conducted using the same weights and probability and possibility levels. Decision makers would be able to fine tune this parameter to obtain different solutions using different levels. The solutions in Table 9 reflect the different optimistic-pessimistic attitudes for uncertainty, where $\lambda=1$ and $\lambda=0$ are the pessimistic extreme and optimistic extreme respectively. Since the purpose of this paper is the minimization of the total cost, transportation duration and total waste, so based on Eqn (1), it can be seen that if the optimistic-pessimistic $\lambda$ rises, the total cost, duration and total waste also gradually increase, which indicates that a more optimistic attitude by the decision maker leads to a more optimistic optimization for the construction pro- ject total cost, duration and total waste. These results are quite useful as a reference for decision makers in planning a dynamic transportation assignment problem. The optimistic-pessimistic index is provided by the decision makers and is interpreted according to the real world problem.

\subsubsection{Sensitivity analysis for the probability and possibility levels}

As shown above, all probability levels are set at the same value $\sigma$ and all possibility levels are set at the same value $\gamma$. By reviewing the method of dealing with the fuzzy random variables, it can be seen that under different probability and possibility levels the objective function values are different. In order to gain insight into the selection principle of the parameters including the optimistic-pessimistic index (i.e. 
Table 5. Data information of transportation waste in each route at each stage

\begin{tabular}{|c|c|c|c|c|}
\hline \multirow{2}{*}{\multicolumn{2}{|c|}{ Model parameters }} & \multicolumn{3}{|c|}{ Stage index } \\
\hline & & $k=1$ & $k=2$ & $k=3$ \\
\hline \multirow{16}{*}{$\begin{array}{c}\tilde{\bar{w}}_{i j}(k) \\
\left(\mathrm{m}^{3} / \text { unit) }\right.\end{array}$} & \multirow{2}{*}{$\tilde{\bar{w}}_{11}(k)$} & $(0.028, \varphi(\omega), 0.040)$ & $(0.032, \varphi(\omega), 0.042)$ & $(0.028, \varphi(\omega), 0.038)$ \\
\hline & & $\varphi(\omega) \sim N(0.034,0.016)$ & $\varphi(\omega) \sim N(0.037,0.02)$ & $\varphi(\omega) \sim N(0.032,0.018)$ \\
\hline & \multirow{2}{*}{$\tilde{\bar{w}}_{21}(k)$} & $(0.020, \varphi(\omega), 0.029)$ & $(0.023, \varphi(\omega), 0.029)$ & $(0.019, \varphi(\omega), 0.026)$ \\
\hline & & $\varphi(\omega) \sim N(0.025,0.018)$ & $\varphi(\omega) \sim N(0.027,0.02)$ & $\varphi(\omega) \sim N(0.022,0.010)$ \\
\hline & \multirow{2}{*}{$\tilde{\overline{\bar{w}}}_{31}(k)$} & $(0.020, \varphi(\omega), 0.029)$ & $(0.018, \varphi(\omega), 0.026)$ & $(0.016, \varphi(\omega), 0.023)$ \\
\hline & & $\varphi(\omega) \sim N(0.023,0.015)$ & $\varphi(\omega) \sim N(0.021,0.016)$ & $\varphi(\omega) \sim N(0.020,0.010)$ \\
\hline & \multirow{2}{*}{$\tilde{\bar{w}}_{41}(k)$} & $(0.036, \varphi(\omega), 0.047)$ & $(0.042, \varphi(\omega), 0.047)$ & $(0.036, \varphi(\omega), 0.045)$ \\
\hline & & $\varphi(\omega) \sim N(0.041,0.023)$ & $\varphi(\omega) \sim N(0.044,0.022)$ & $\varphi(\omega) \sim N(0.040,0.030)$ \\
\hline & \multirow{2}{*}{$\tilde{\bar{w}}_{12}(k)$} & $(0.022, \varphi(\omega), 0.031)$ & $(0.025, \varphi(\omega), 0.035)$ & $(0.019, \varphi(\omega), 0.025)$ \\
\hline & & $\varphi(\omega) \sim N(0.028,0.018)$ & $\varphi(\omega) \sim N(0.030,0.016)$ & $\varphi(\omega) \sim N(0.023,0.012)$ \\
\hline & \multirow{2}{*}{$\tilde{\overline{\mathcal{w}}}_{22}(k)$} & $(0.019, \varphi(\omega), 0.026)$ & $(0.019, \varphi(\omega), 0.029)$ & $(0.018, \varphi(\omega), 0.025)$ \\
\hline & & $\varphi(\omega) \sim N(0.022,0.014)$ & $\varphi(\omega) \sim N(0.025,0.012)$ & $\varphi(\omega) \sim N(0.021,0.011)$ \\
\hline & \multirow{2}{*}{$\tilde{\bar{w}}_{32}(k)$} & $(0.038, \varphi(\omega), 0.043)$ & $(0.037, \varphi(\omega), 0.047)$ & $(0.032, \varphi(\omega), 0.041)$ \\
\hline & & $\varphi(\omega) \sim N(0.041,0.023)$ & $\varphi(\omega) \sim N(0.043,0.022)$ & $\varphi(\omega) \sim N(0.038,0.025)$ \\
\hline & \multirow{2}{*}{$\tilde{\bar{w}}_{42}(k)$} & $(0.027, \varphi(\omega), 0.036)$ & $(0.027, \varphi(\omega), 0.039)$ & $(0.022, \varphi(\omega), 0.035)$ \\
\hline & & $\varphi(\omega) \sim N(0.032,0.018)$ & $\varphi(\omega) \sim N(0.035,0.023)$ & $\varphi(\omega) \sim N(0.029,0.022)$ \\
\hline \multirow{4}{*}{$\begin{array}{c}\tilde{\bar{w}}_{j j}(k) \\
\left(\mathrm{m}^{3} / \text { unit) }\right.\end{array}$} & \multirow{2}{*}{$\tilde{\bar{w}}_{11}(k)$} & $(0.028, \varphi(\omega), 0.037)$ & $(0.031, \varphi(\omega), 0.043)$ & $(0.026, \varphi(\omega), 0.038)$ \\
\hline & & $\varphi(\omega) \sim N(0.032,0.018)$ & $\varphi(\omega) \sim N(0.037,0.021)$ & $\varphi(\omega) \sim N(0.029,0.022)$ \\
\hline & \multirow{2}{*}{$\tilde{\bar{w}}_{22}(k)$} & $(0.016, \varphi(\omega), 0.026)$ & $(0.018, \varphi(\omega), 0.028)$ & $(0.016, \varphi(\omega), 0.025)$ \\
\hline & & $\varphi(\omega) \sim N(0.021,0.012)$ & $\varphi(\omega) \sim N(0.024,0.014)$ & $\varphi(\omega) \sim N(0.020,0.011)$ \\
\hline
\end{tabular}

$\lambda$ ), probability levels (i.e. $\sigma$ ) and possibility levels (i.e. $\gamma$ ), a sensitivity analysis was conducted against these parameters. Table 10 summarizes the different total fitness values with respect to the different parameters $\lambda, \sigma$ and $\gamma$.

These are summarized as follows:

(1)For the probability level $\sigma$, parameters $\lambda, \eta_{c}, \eta_{d}, \eta_{w}$ and possibility level $\gamma$ are all fixed, when $\lambda<0.5$, the bigger $\sigma$, the bigger the objective function values; when $\lambda>0.5$, the bigger $\sigma$, the smaller the objective function values.

(2)For the possibility level $\gamma$, parameters $\lambda, \eta_{c}, \eta_{d}, \eta_{w}$ and probability level $\sigma$ are all fixed, when $\lambda<0.5$, the bigger $\gamma$, the smaller the objective function values; when $\lambda>0.5$, the bigger $\gamma$, the bigger the objective function values.
These results are quite useful and may serve as references for decision-makers and in fact, it would be their choice to identify an appropriate set of parameter values (i.e. $\lambda, \sigma$ and $\gamma$ ) to optimize the decision making process.

\subsubsection{Sensitivity analysis for the weights of objective functions}

From the discussion above, it can be seen that a difference in weights leads to a difference in objective function values. The results are shown in Table 11 with respect to different weights, in which the optimisticpessimistic index $\lambda=0.5$, probability level $\sigma=0.2$, possibility level $\gamma=0.8$. These comparative results demonstrate that the difference in solutions with different weights is not very big, because the weights reflect 
Table 6. Data information of excavation price, storable price and demand of each filling area at each stage

\begin{tabular}{|c|c|c|c|c|}
\hline \multicolumn{2}{|c|}{ Model parameters } & \multicolumn{3}{|c|}{ Stage index } \\
\hline & & $k=1$ & $k=2$ & $k=3$ \\
\hline \multirow{8}{*}{$\begin{array}{c}\tilde{\bar{c}}_{i}(k) \\
\text { (CNY/unit) }\end{array}$} & \multirow{2}{*}{$\tilde{\bar{c}}_{1}(k)$} & $(14.82, \varphi(\omega), 15.56)$ & $(15.26, \varphi(\omega), 16.44)$ & $(16.28, \varphi(\omega), 17.18)$ \\
\hline & & $\varphi(\omega) \sim N(15.20,12.2)$ & $\varphi(\omega) \sim N(16.00,13.0)$ & $\varphi(\omega) \sim N(16.82,13.8)$ \\
\hline & \multirow{2}{*}{$\tilde{\bar{c}}_{2}(k)$} & $(14.02, \varphi(\omega), 14.97)$ & $(13.23, \varphi(\omega), 14.09)$ & $(14.18, \varphi(\omega), 15.25)$ \\
\hline & & $\varphi(\omega) \sim N(14.55,12.0)$ & $\varphi(\omega) \sim N(13.66,12.1)$ & $\varphi(\omega) \sim N(14.72,12.8)$ \\
\hline & \multirow{2}{*}{$\tilde{\bar{c}}_{3}(k)$} & $(14.89, \varphi(\omega), 15.67)$ & $(13.97, \varphi(\omega), 14.55)$ & $(15.16, \varphi(\omega), 15.83)$ \\
\hline & & $\varphi(\omega) \sim N(15.23,13.2)$ & $\varphi(\omega) \sim N(14.34,11.0)$ & $\varphi(\omega) \sim N(15.59,11.9)$ \\
\hline & \multirow{2}{*}{$\tilde{\bar{c}}_{4}(k)$} & $(15.78, \varphi(\omega), 16.47)$ & $(16.04, \varphi(\omega), 16.58)$ & $(16.46, \varphi(\omega), 17.45)$ \\
\hline & & $\varphi(\omega) \sim N(16.12,13.5)$ & $\varphi(\omega) \sim N(16.44,13.2)$ & $\varphi(\omega) \sim N(16.80,14.0)$ \\
\hline \multirow{4}{*}{$\begin{array}{c}\tilde{\bar{c}}_{j}^{1}(k) \\
\text { (CNY/unit) }\end{array}$} & \multirow{2}{*}{$\tilde{\bar{c}}_{1}^{1}(k)$} & $(1.00, \varphi(\omega), 1.32)$ & $(1.10, \varphi(\omega), 1.44)$ & \\
\hline & & $\varphi(\omega) \sim N(1.14,1.0)$ & $\varphi(\omega) \sim N(1.27,1.1)$ & \\
\hline & \multirow{2}{*}{$\tilde{\bar{c}}_{2}^{1}(k)$} & $(0.91, \varphi(\omega), 1.57)$ & $(1.23, \varphi(\omega), 1.69)$ & \\
\hline & & $\varphi(\omega) \sim N(1.25,1.1)$ & $\varphi(\omega) \sim N(1.36,1.2)$ & \\
\hline \multirow{4}{*}{$\begin{array}{c}\tilde{\bar{c}}_{j}^{2}(k) \\
(\mathrm{CNY} / \text { unit) }\end{array}$} & \multirow{2}{*}{$\tilde{\bar{c}}_{1}^{2}(k)$} & $(0.40, \varphi(\omega), 0.60)$ & $(0.52, \varphi(\omega), 0.68)$ & $(0.48, \varphi(\omega), 0.62)$ \\
\hline & & $\varphi(\omega) \sim N(0.50,0.40)$ & $\varphi(\omega) \sim N(0.60,0.49)$ & $\varphi(\omega) \sim N(0.56,0.36)$ \\
\hline & \multirow{2}{*}{$\tilde{\bar{c}}_{2}^{2}(k)$} & $(0.36, \varphi(\omega), 0.45)$ & $(0.50, \varphi(\omega), 0.60)$ & $(0.40, \varphi(\omega), 0.50)$ \\
\hline & & $\varphi(\omega) \sim N(0.40,0.25)$ & $\varphi(\omega) \sim N(0.56,0.36)$ & $\varphi(\omega) \sim N(0.42,0.49)$ \\
\hline \multirow{2}{*}{$\begin{array}{c}\bar{d}_{j}(k) \\
\left(10^{4} \mathrm{~m}^{3}\right)\end{array}$} & \multirow{2}{*}{$\begin{array}{l}\bar{d}_{1}(k) \\
\bar{d}_{2}(k)\end{array}$} & $(364.6,368.2,371.1)$ & $(424.2,426.6,428.9)$ & $(373.1,374.5,376.7)$ \\
\hline & & $(375.6,377.1,379.8)$ & $(447.3,449.4,452.1)$ & $(370.0,372.3,373.8)$ \\
\hline \multirow{4}{*}{$\begin{array}{c}\tilde{\bar{c}}_{j}(k) \\
\text { (CNY/unit) }\end{array}$} & \multirow{2}{*}{$\tilde{\bar{c}}_{1}(k)$} & $(0.42, \varphi(\omega), 0.55)$ & $(0.50, \varphi(\omega), 0.065)$ & $(0.48, \varphi(\omega), 60)$ \\
\hline & & $\varphi(\omega) \sim N(0.50,0.36)$ & $\varphi(\omega) \sim N(0.038,0.025)$ & $\varphi(\omega) \sim N(0.54,0.040)$ \\
\hline & \multirow{2}{*}{$\tilde{\bar{c}}_{2}(k)$} & $(0.36, \varphi(\omega), 0.46)$ & $(0.48, \varphi(\omega), 0.60)$ & $(0.40, \varphi(\omega), 0.52)$ \\
\hline & & $\varphi(\omega) \sim N(0.40,0.25)$ & $\varphi(\omega) \sim N(0.52,0.38)$ & $\varphi(\omega) \sim N(0.48,0.30)$ \\
\hline \multirow{2}{*}{$\begin{array}{c}h_{j}^{k}(\bar{t}) \\
\left(10^{4} \mathrm{~m}^{3}\right)\end{array}$} & $h_{1}^{k}(\bar{t})$ & 25 & 23 & 22 \\
\hline & $h_{2}^{k}(\bar{t})$ & 24 & 22 & 23 \\
\hline
\end{tabular}

Table 7. Parameter selection for DP-based CPSO

\begin{tabular}{cccccc}
\hline $\begin{array}{c}\text { Population } \\
\text { size }\end{array}$ & $\begin{array}{c}\text { Iteration } \\
\text { number }\end{array}$ & \multicolumn{2}{c}{$\begin{array}{c}\text { Acceleration } \\
\text { constant }\end{array}$} & Iteration weight \\
\hline$L$ & $T$ & $c_{p}$ & $c_{g}$ & $w(1)$ & $w(T)$ \\
\hline 100 & 100 & 2 & 2 & 0.9 & 0.1 \\
\hline
\end{tabular}

the importance of each objective from the view of decision makers. Therefore, the results become gradually worse with an increase in the importance of the objective function $f_{w}$. However, in a real situation, decision makers would control the weights within a reasonable range and they would be interpreted according to the real world problem. 
Table 8. Results of DP-based CPSO Algorithm for TS-based DTAP $\left(\lambda=0.5, \eta_{c}=0.5, \eta_{w}=0.3, \sigma=0.2, \gamma=0.8\right)$

\begin{tabular}{lcccccc}
\hline \multirow{2}{*}{ Objective } & \multicolumn{3}{c}{ Total construction cost, duration and waste } & Convergence & Computation \\
\cline { 2 - 6 } & Fitness value & Actual data & Net decrease & Rate of decrease & iteration number & time $(\mathrm{s})$ \\
\hline$f$ & 0.8773 & 0.9220 & 0.0379 & $4.47 \%$ & 46 & 17.34 \\
\hline$f_{c}\left(10^{6}\right)$ & 582.50 & 594.74 & 12.24 & $2.06 \%$ & 46 & 17.34 \\
\hline$f_{d}\left(10^{1}\right)$ & 244.47 & 262.36 & 17.89 & $6.82 \%$ & 46 & 17.34 \\
\hline$f_{w}\left(10^{4}\right)$ & 154.16 & 170.06 & 15.90 & $9.35 \%$ & 46 & 17.34 \\
\hline
\end{tabular}

Table 9. Sensitivity analysis on the optimistic-pessimistic index $\lambda$ of decision makers $\left(\eta_{c}=0.5, \eta_{d}=0.3, \eta_{w}=0.2, \sigma=0.2, \gamma=0.8\right)$

\begin{tabular}{|c|c|c|c|c|c|c|c|c|c|c|c|}
\hline \multirow{2}{*}{ Objective } & \multicolumn{11}{|c|}{ Optimistic-pessimistic index } \\
\hline & $\lambda=0$ & $\lambda=0.1$ & $\lambda=0.2$ & $\lambda=0.3$ & $\lambda=0.4$ & $\lambda=0.5$ & $\lambda=0.6$ & $\lambda=0.7$ & $\lambda=0.8$ & $\lambda=0.9$ & $\lambda=0.10$ \\
\hline$f$ & 0.8173 & 0.8294 & 0.8402 & 0.8543 & 0.8664 & 0.8773 & 0.8887 & 0.9008 & 0.9132 & 0.9250 & 0.9371 \\
\hline$f_{c}\left(10^{6}\right)$ & 566.93 & 570.08 & 573.17 & 576.29 & 579.41 & 582.50 & 585.61 & 588.73 & 591.86 & 594.98 & 598.12 \\
\hline$f_{d}\left(10^{1}\right)$ & 216.30 & 221.78 & 226.36 & 234.12 & 239.45 & 244.47 & 249.37 & 254.52 & 260.28 & 265.85 & 271.31 \\
\hline$f_{w}\left(10^{4}\right)$ & 136.49 & 140.25 & 143.76 & 147.12 & 151.03 & 154.16 & 157.87 & 162.02 & 165.81 & 169.22 & 173.01 \\
\hline
\end{tabular}

Table 10. Sensitivity analysis on the probability level $\sigma$ and possibility level $\gamma$ of decision makers

\begin{tabular}{|c|c|c|c|c|c|c|c|c|c|c|c|c|c|}
\hline \multirow{2}{*}{$\sigma$} & \multirow{2}{*}{ Objective } & \multicolumn{3}{|c|}{$\lambda=0$} & \multicolumn{3}{|c|}{$\lambda=0.25$} & \multicolumn{3}{|c|}{$\lambda=0.75$} & \multicolumn{3}{|c|}{$\lambda=1$} \\
\hline & & $\gamma=0.7$ & $\gamma=0.8$ & $\gamma=0.9$ & $\gamma=0.7$ & $\gamma=0.8$ & $\gamma=0.9$ & $\gamma=0.7$ & $\gamma=0.8$ & $\gamma=0.9$ & $\gamma=0.7$ & $\gamma=0.8$ & $\gamma=0.9$ \\
\hline \multirow[t]{4}{*}{0.2} & $f$ & 0.829 & 0.817 & 0.805 & 0.859 & 0.847 & 0.837 & 0.896 & 0.907 & 0.914 & 0.926 & 0.937 & 0.949 \\
\hline & $f_{c}\left(10^{6}\right)$ & 569.9 & 566.9 & 562.5 & 578.3 & 574.7 & 571.9 & 586.7 & 590.1 & 593.1 & 594.9 & 598.1 & 601.5 \\
\hline & $f_{d}\left(10^{1}\right)$ & 221.1 & 216.3 & 211.8 & 234.9 & 230.3 & 225.5 & 253.2 & 257.8 & 262.7 & 266.2 & 271.3 & 276.3 \\
\hline & $f_{w}\left(10^{4}\right)$ & 140.1 & 136.5 & 132.2 & 148.9 & 145.4 & 142.2 & 160.1 & 163.9 & 163.4 & 169.6 & 173 & 176.6 \\
\hline \multirow[t]{4}{*}{0.4} & $f$ & 0.838 & 0.828 & 0.817 & 0.870 & 0.858 & 0.847 & 0.886 & 0.896 & 0.908 & 0.916 & 0.927 & 0.938 \\
\hline & $f_{c}\left(10^{6}\right)$ & 572.5 & 569.8 & 566.1 & 581.8 & 577.9 & 574.2 & 583.4 & 587.2 & 590.9 & 591.6 & 594.9 & 598.2 \\
\hline & $f_{d}\left(10^{1}\right)$ & 225.4 & 220.8 & 216.5 & 239.7 & 234.7 & 230.2 & 249.0 & 253.1 & 258.0 & 262.2 & 266.7 & 271.5 \\
\hline & $f_{w}\left(10^{4}\right)$ & 143.3 & 140.2 & 136.7 & 152.3 & 149.1 & 146.1 & 157.1 & 160.2 & 164.2 & 166.3 & 170.1 & 173.5 \\
\hline \multirow[t]{4}{*}{0.6} & $f$ & 0.850 & 0.840 & 0.829 & 0.880 & 0.868 & 0.858 & 0.875 & 0.886 & 0.897 & 0.904 & 0.915 & 0.926 \\
\hline & $f_{c}\left(10^{6}\right)$ & 575.9 & 572.6 & 569.4 & 584.2 & 581.0 & 577.4 & 580.0 & 584.7 & 587.7 & 588.3 & 591.5 & 594.8 \\
\hline & $f_{d}\left(10^{1}\right)$ & 230.1 & 225.5 & 221.3 & 244.2 & 238.9 & 234.5 & 244.2 & 248.7 & 253.4 & 257.1 & 261.8 & 266.5 \\
\hline & $f_{w}\left(10^{4}\right)$ & 147.1 & 144.2 & 140.3 & 155.5 & 152.3 & 149.7 & 154.1 & 157.1 & 160.4 & 163.2 & 166.4 & 170.1 \\
\hline \multirow[t]{4}{*}{0.8} & $f$ & 0.860 & 0.850 & 0.840 & 0.890 & 0.880 & 0.870 & 0.864 & 0.875 & 0.887 & 0.893 & 0.904 & 0.916 \\
\hline & $f_{c}\left(10^{6}\right)$ & 578.2 & 575.9 & 572.5 & 587.5 & 584.3 & 581.5 & 577.7 & 580.0 & 584.4 & 584.9 & 588.2 & 591.6 \\
\hline & $f_{d}\left(10^{1}\right)$ & 234.9 & 230.2 & 225.9 & 248.9 & 243.9 & 239.4 & 239.6 & 243.9 & 248.4 & 251.7 & 256.7 & 261.7 \\
\hline & $f_{w}\left(10^{4}\right)$ & 150.5 & 147.3 & 144.2 & 158.6 & 155.6 & 152.8 & 150.5 & 154.2 & 158.1 & 159.9 & 163.3 & 166.9 \\
\hline
\end{tabular}

\subsubsection{Model comparison in different environments}

To indicate and highlight the superiority of usage for the fuzzy random variables for the mathematical model (15) in this paper, additional computational work was done using the proposed DP-based CPSO to solve a similar a similar TS-based DTAP model under the other two environments (i.e. determined environment and fuzzy environment). To carry out comparisons under a similar circumstance, analysis was conducted based on results from running the test problem many times. A detailed analysis follows.

In order to guarantee a fair comparison between the TS-based DTAP model under a fuzzy random environ- ment and a similar TS-based DTAP model under other environments, each related uncertain parameter in the TS-based DTAP was selected in the following way. As shown in section "Key problem statement", denote the fuzzy random variables as $\tilde{\bar{\xi}}=\left([\xi]_{L}, \varphi(\omega),[\xi]_{R}\right)$, where $\varphi(\omega)$ is a random variable with following a Normal distribution $N\left(\mu, \eta^{2}\right)$. Since the variance of $\varphi(\omega)$ was sufficiently small, and the expectation value $\mu$ essentially reflected the most possible value over time. Thus, it was reasonable to select $\left([\xi]_{L}, \mu,[\xi]_{R}\right)$ and $\mu$ as the fuzzy parameter and certain parameter for a fuzzy environment 
Table 11. Sensitivity analysis on the weights $\eta_{c}, \eta_{d}$, and $\eta_{w}$ of decision makers $(\lambda=0.5, \sigma=0.2, \gamma=0.8)$

\begin{tabular}{|c|c|c|c|c|c|c|c|}
\hline \multirow{2}{*}{ Combinations } & \multicolumn{3}{|c|}{ Weights } & \multicolumn{4}{|c|}{ Objective values } \\
\hline & $\eta_{c}$ & $\eta_{d}$ & $\eta_{w}$ & $f$ & $f_{c}\left(10^{6}\right)$ & $f_{d}\left(10^{1}\right)$ & $f_{w}\left(10^{4}\right)$ \\
\hline Combination 1 & 0.6 & 0.2 & 0.2 & 0.8814 & 574.92 & 247.30 & 156.45 \\
\hline Combination 2 & 0.5 & 0.3 & 0.2 & 0.8773 & 582.50 & 244.47 & 154.16 \\
\hline Combination 3 & 0.5 & 0.2 & 0.3 & 0.8707 & 584.35 & 249.63 & 151.18 \\
\hline Combination 4 & 0.4 & 0.4 & 0.2 & 0.8647 & 590.26 & 237.20 & 153.85 \\
\hline Combination 5 & 0.4 & 0.3 & 0.3 & 0.8598 & 591.70 & 241.53 & 150.24 \\
\hline Combination 6 & 0.4 & 0.2 & 0.4 & 0.8500 & 590.50 & 248.25 & 147.53 \\
\hline
\end{tabular}

and a determined environment respectively. Thus the models for the similar TS-based DTAP under different environments were formulated and solved using the proposed DP-based CPSO, the computational results were obtained based on weights $\eta_{c}=0.5, \eta_{d}=0.3$ and $\eta_{w}=$ 0.2 as shown in Table 12. By comparing the fitness value of the three objectives and the aggregated objective, the results for the discussed two types for the TS-based DTAP are higher than the fuzzy random type, our findings highlight the superiority of usage for the fuzzy random variables for the TS-based DTAP model proposed in this paper, and also indicate that TS-based DTAP model using fuzzy set theory has a much better performance than using certain parameters. The performance also suggests that DP-based CPSO is an effective and relatively efficient approach for solving the TS-based DTAP model.

\subsubsection{Algorithm evaluation}

To carry out comparisons under a similar circumstance, the parameters stated in Table 5 and the initial velocities for the decision variables in the DP-based CPSO are also adopted for the standard PSO. However, the initial velocities for the state variables in the standard PSO are selected based on the inventory level of the borrow areas and stockpile areas at each stage. Table 13 shows the comparison results and the convergence histories of the DP-based CPSO and standard PSO respectively based on an optimistic-pessimistic index $\lambda=0.5$, weights $\eta_{c}=$ $0.5, \eta_{d}=0.3$ and $\eta_{w}=0.2$, probability level $\sigma=0.2$ and possibility level $\gamma=0.8$. The DP-based CPSO proposed in this paper has an obvious advantage compared with the standard PSO when solving TS-based DTAP. The first advantage is that the DP-based CPSO is more stable than a standard PSO when searching for the op-

Table 12. Model comparison in different environments

\begin{tabular}{lcccc}
\hline \multirow{2}{*}{ Environments } & \multicolumn{4}{c}{ Objective function values } \\
\cline { 2 - 5 } & $f$ & $f_{c}\left(10^{6}\right)$ & $f_{d}\left(10^{1}\right)$ & $f_{w}\left(10^{4}\right)$ \\
\hline $\begin{array}{l}\text { Determined } \\
\text { environment }\end{array}$ & 0.9077 & 589.67 & 256.81 & 165.72 \\
\hline $\begin{array}{l}\text { Fuzzy } \\
\text { environment }\end{array}$ & 0.8862 & 584.23 & 248.65 & 157.26 \\
\hline $\begin{array}{l}\text { Fuzzy random } \\
\text { environment }\end{array}$ & 0.8773 & 582.50 & 244.47 & 154.16 \\
\hline
\end{tabular}

tima. Another is that the proposed DP-based CPSO is faster when determining the optima and converges a little faster than the standard PSO, that is, the DP-based CPSO needs less iterations to find the optimal solutions. Thus the DP-based CPSO displays an improved search performance compared with the standard PSO under a similar circumstance.

\section{Conclusions and future research}

In this paper, a multistage multi-objective optimal control expected value model is established to solve a TS-based DTAP in an earth-rock transportation system under a fuzzy random environment. It is a multi-objective dynamic programming optimization process for minimizing total operational cost, transportation duration and total waste. Dump truck and belt conveyors characterize the features of the two-stage based earth-rock transportation system and construction site environment. Decision-makers determine a suitable resource allocation and route assignment to dynamically assign the transport between the different transportation subsystems to minimize the objectives. While using probability theory is cumbersome and costly, and fuzzy theory is incapable of dealing with ambiguous and complex information, triangular fuzzy random variables are used to characterize the multiple parameter uncertainties with combinations of both fuzziness and random-

Table 13. Comparison results between DP-based CPSO and Standard PSO

\begin{tabular}{lcccc}
\hline Approach & $\begin{array}{c}\text { Objective } \\
\text { functions }\end{array}$ & $\begin{array}{c}\text { Minimal } \\
\text { fitness } \\
\text { value }\left(10^{6}\right)\end{array}$ & $\begin{array}{c}\text { Iterations } \\
\text { to find } \\
\text { optima }\end{array}$ & $\begin{array}{c}\text { Computation } \\
\text { time(s) }\end{array}$ \\
\hline $\begin{array}{l}\text { DP-based } \\
\text { PSO }\end{array}$ & $f$ & 0.8773 & 46 & 17.34 \\
\hline & $f_{c}\left(10^{6}\right)$ & 582.50 & 46 & 17.34 \\
\hline & $f_{d}\left(10^{1}\right)$ & 244.47 & 46 & 17.34 \\
\hline & $f_{w}\left(10^{4}\right)$ & 154.16 & 46 & 17.34 \\
\hline $\begin{array}{l}\text { Standard } \\
\text { PSO }\end{array}$ & $f$ & 0.8773 & 74 & 32.20 \\
\hline & $f_{c}\left(10^{6}\right)$ & 582.50 & 74 & 32.20 \\
\hline & $f_{d}\left(10^{1}\right)$ & 244.47 & 74 & 32.20 \\
\hline & $f_{w}\left(10^{4}\right)$ & 154.16 & 74 & 32.20 \\
\hline
\end{tabular}


ness. A hybrid crisp approach and an expected value operator are introduced to deal with these uncertainties. This method first transforms the fuzzy random parameters into $(\gamma, \sigma)$ - level trapezoidal fuzzy variables, which are subsequently defuzzified using an expected value operator with an optimistic-pessimistic index. Thus, the expected value model is derived. This paper's objectives are to comprehensively consider the multipleobjectives, the double multiple-stages, the dynamic factors, and the hybrid uncertainty. Subsequently, to solve the above problem, a multi-objective DP-based CPSO algorithm was developed based on the particular nature of the model, which is able to automatically control the particle-updating in the feasible solution space to find the optimal solution for the expected value model. Finally, an earth-rock dam construction at the Pubugou Hydropower project was used as a practical application example to verify the proposed approach. The results and analysis are presented to highlight the performance of our optimization method, which was proven to have the characteristics of generality, less calculation, high velocity, high efficiency and high precision compared to the standard PSO algorithm.

It should be noted that our TS-based DTAP excepted value model is formulated with some assumptions, so it may not represent the precise construction and transportation environment. With this in mind, an important area for future research would be the consideration of more restrictions rather than assumptions. For example, by balancing the allocation of earth-rock work from the borrow areas, decision-makers usually add a series of restrictions, which would be beneficial in maintaining the stability of the supply chain. Another area of improvement would be the consideration of including more details about the stockpile areas and the changing unit storage cost over time. Therefore, more research needs to be done and evidence gathered in future research to find solutions to the above problems and develop a more efficient heuristic method to derive modified solutions.

\section{Acknowledgements}

This research was supported by the Key Program of National Natural Science Foundation of China (Grant No. 70831005), the Research Foundation of Ministry of Education for the Doctoral Program of Higher Education of China (Grant No. 20130181110063) and "985" Program of Sichuan University (Innovative Research Base for Economic Development and Management).

\section{References}

Ali, A. M.; Sik, Y. H. 2012. Transportation problem: a special case for linear programing problems in mining engineering, International Journal of Mining Science and Technology 22(3): 371-377. http://dx.doi.org/10.1016/j.ijmst.2012.04.015

Ammar, E. E 2008. On solutions of fuzzy random multiobjective quadratic programming with applications in portfolio problem, Information Sciences 178(2): 468-484. http://dx.doi.org/10.1016/j.ins.2007.03.029
Anghinolfi, D.; Paolucci, M. 2009. A new discrete particle swarm optimization approach for the single-machine total weighted tardiness scheduling problem with sequencedependent setup times, European Journal of Operational Research 193(1): 73-85.

http://dx.doi.org/10.1016/j.ejor.2007.10.044

Barbarosoglu, G.; Arda, Y. 2004. A two-stage stochastic programming framework for transportation planning in disaster response, Journal of the Operational Research Society 55: 43-53. http://dx.doi.org/10.1057/palgrave.jors.2601652

Bell, J. E.; McMullen, P. R. 2004. Ant colony optimization techniques for the vehicle routing problem, Advanced Engineering Informatics 18(1): 41-48. http://dx.doi.org/10.1016/j.aei.2004.07.001

Bliemer, M. C. J.; Bovy, P. H. L. 2003. Quasi-variational inequality formulation of the multiclass dynamic traffic assignment problem, Transportation Research Part B: Methodological 37(6): 501-519.

http://dx.doi.org/10.1016/S0191-2615(02)00025-5

Bojadziev, G.; Bojadziev, M. 1997. Fuzzy logic for business, finance, and management. $1^{\text {st }}$ ed. Singapore: World Scientific. 232 p.

Cao, S. H.; Yuan, Z. Z.; Li, Y. H.; Wu, X. Y. 2007. Model for road network stochastic user equilibrium based on bi-level programming under the action of the traffic flow guidance system, Journal of Transportation Systems Engineering and Information Technology 7(4): 36-42. http://dx.doi.org/10.1016/S1570-6672(07)60028-2

Cascetta, E. 1989. A stochastic process approach to the analysis of temporal dynamics in transportation networks, Transportation Research Part B: Methodological 23(1): 1-17. http://dx.doi.org/10.1016/0191-2615(89)90019-2

Celik, M.; Cebi, S.; Kahraman, C.; Er, I. D. 2009. Application of axiomatic design and TOPSIS methodologies under fuzzy environment for proposing competitive strategies on Turkish container ports in maritime transportation network, Expert Systems with Applications 36(3): 4541-4557.

Chanas, S.; Kolodziejczyk, W.; Machaj, A. 1984. A fuzzy approach to the transportation problem, Fuzzy Sets and Systems 13(3): 211-221.

http://dx.doi.org/10.1016/0165-0114(84)90057-5

Chanas, S.; Kuchta, D. 1996. A concept of the optimal solution of the transportation problem with fuzzy cost coefficients, Fuzzy Sets and Systems 82(3): 299-305. http://dx.doi.org/10.1016/0165-0114(95)00278-2

Clementi, A. E. F.; Ianni, M. D.; Silvestri, R. 2003. The minimum broadcast range assignment problem on linear multi-hop wireless networks, Theoretical Computer Science 299(1-3): 751-761. http://dx.doi.org/10.1016/S0304-3975(02)00538-8

Clerc, M.; Kennedy, J. 2002. The particle swarm: explosion, stability, and convergence in a multidimensional complex space, IEEE Transactions on Evolutionary Computation 6(1): 58-73.

Crainic, T. G.; Ricciardi, N.; Storchi, G. 2004. Advanced freight transportation systems for congested urban areas, Transportation Research Part C: Emerging Technologies 12(2): 119-137.

De Meo, P.; Quattrone, G.; Ursino, D. 2008. A decision support system for designing new services tailored to citizen profiles in a complex and distributed e-government scenario, Data Knowledge Engineering 67(1): 161-184.

Doerner, K.; Hartl, R. F.; Reimann, M. 2001. Cooperative ant colonies for optimizing resource allocation in transportation, Applications of Evolutionary Computing Lecture Notes in Computer Science 2037: 70-79.

Dubois, D.; Prade, H. 1978. Operations on fuzzy numbers, International Journal of System Sciences 9(6): 613-626. http://dx.doi.org/10.1080/00207727808941724 
Dutta, P.; Chakraborty, D.; Roy, A. R. 2005. A single-period inventory model with fuzzy random variable demand, Mathematical and Computer Modelling 41(8-9): 915-922. http://dx.doi.org/10.1016/j.mcm.2004.08.007

Ebben, M.; Zee van der, D. J.; Heijden van der, M. 2004. Dynamic one-way traffic control in automated transportation systems, Transportation Research Part B: Methodological 38(5): 441-458. http://dx.doi.org/10.1016/S0191-2615(03)00075-4

Eberhart, R. C.; Shi, Y. 2001. Tracking and optimizing dynamic systems with particle swarms, in Proceedings of the IEEE Congress on Evolutionary Computation, 27-30 May 2001, Seoul, Korea, 94-97.

El-Wahed, W. F. A.; Lee, S. M. 2006. Interactive fuzzy goal programming for multi-objective transportation problems, Omega 34(2): 158-166.

Friesz, T. L.; Anandalingam, G.; Mehta, N. J.; Nam, K.; Shah, S. J.; Tobin, R. L. 1993. The multiobjective equilibrium network design problem revisited: a simulated annealing approach, European Journal of Operational Research 65(1): 44-57. http://dx.doi.org/10.1016/0377-2217(93)90143-B

Gen, M.; Ida, K.; Li, Y. Z. 1998. Bicriteria transportation problem by hybrid genetic algorithm, Computers \& Industrial Engineering 35(1-2): 363-366.

Gil, M. A.; Lopez-Diaz, M.; Ralescu, D. A. 2006. Overview on the development of fuzzy random variables, Fuzzy Sets and Systems 157(19): 2546-2557. http://dx.doi.org/10.1016/j.fss.2006.05.002

Hurink, J. L.; Knust, S. 2001. Makespan minimization for flowshop problems with transportation times and a single robot, Discrete Applied Mathematics 112(1-3): 199-216.

Islam, S.; Roy, T. K. 2006. A new fuzzy multi-objective programming: entropy based geometric programming and its application of transportation problems, European Journal of Operational Research 173(2): 387-404.

Kennedy, J.; Eberhart, R. 1995. Particle swarm optimization, in Proceedings of the IEEE International Conference on Neural Networks, 27 November - 01 December 1995, Perth, WA, 1942-1948.

Kruse, R.; Meyer, K. D. 1987. Statistics with vague data. $1^{\text {st }}$ ed. D. Reidel Publishing Company. 279 p.

Kwakernaak, H. 1978. Fuzzy random variables. Part I: Definitions and theorems, Information Sciences 15(1): 1-29. http://dx.doi.org/10.1016/0020-0255(78)90019-1

Kwakernaak, H. 1979. Fuzzy random variables. Part II: Algorithms and examples for the discrete case, Information Sciences 17(3): 253-278. http://dx.doi.org/10.1016/0020-0255(79)90020-3

Li, G. Z.; Simha, R. 2000. On the wavelength assignment problem in multifiber WDM star and ring networks, in Nineteenth Annual Joint Conference of the IEEE Computer and Communications Societies, 26-30 March 2000, Tel Aviv, Israel, 1771-1780.

Liu, S. T.; Kao, C. 2004. Solving fuzzy transportation problems based on extension principle, European Journal of Operational Research 153(3): 661-674. http://dx.doi.org/10.1016/S0377-2217(02)00731-2

Luathep, P.; Sumalee, A; Lam, W. H.; Li, Z. C.; Lo, H. K. 2011. Global optimization method for mixed transportation network design problem: a mixed-integer linear programming approach, Transportation Research Part B: Methodological 45(5): 808-827.

Maher, M. J.; Zhang, X. Y.; Van Vliet, D. V. 2001. A bi-level programming approach for trip matrix estimation and traffic control problems with stochastic user equilibrium link flows, Transportation Research Part B: Methodological 35(1): $23-40$.
Michalewicz, Z.; Vignaux, G. A.; Hobbs, M. 1991. A nonstandard genetic algorithm for the nonlinear transportation problem, Journal on Computing 3(4): 307-316. http://dx.doi.org/10.1287/ijoc.3.4.307

Möller, B.; Reuter, U. 2007. Uncertainty forecasting in engineering. Springer. $216 \mathrm{p}$.

Naderi, B.; Zandieh, M.; Khaleghi Ghoshe Balagh, A.; Roshanaei, V. 2009. An improved simulated annealing for hybrid flowshops with sequence-dependent setup and transportation times to minimize total completion time and total tardiness, Expert Systems with Applications 36(6): 9625-9633. http://dx.doi.org/10.1016/j.eswa.2008.09.063

Papadopoulos, H. T. 1996. A field service support system using a queueing network model and the priority MVA algorithm, Omega 24(2): 195-203.

Pardo, M. J.; de la Fuente, D. 2007. Optimizing a priority-discipline queueing model using fuzzy set theory, Computers and Mathematics with Applications 54(2): 267-281. http://dx.doi.org/10.1016/j.camwa.2007.01.019

Peidro, D.; Mula, J.; Poler, R.; Verdegay, J. L. 2009. Fuzzy optimization for supply chain planning under supply, demand and process uncertainties, Decision and Optimisation 160(18): 2640-2657. http://dx.doi.org/10.1016/j.fss.2009.02.021

Puri, M. L.; Ralescu, D. A. 1986. Fuzzy random variables, Journal of Mathematical Analysis and Applications 114(2): 409-422.

Shi, Y.; Eberhart, R. C. 1998. Parameter selection in particle swarm optimization, in Proceedings of the Seventh International Conference on Evolutionary Programming, 25-27 March 1998, San Diego, California, 591-600.

Shu, J.; Teo, C. P.; Shen, Z. M. 2005. Stochastic transportationinventory network design problem, Operations Research 53(1): 48-60. http://dx.doi.org/10.1287/opre.1040.0140

Sumalee, A.; Watling, D. P.; Nakayama, S. 2006. Reliable network design problem: case with uncertain demand and total travel time reliability, Journal of the Transportation Research Board 1964(1): 81-90.

Teodorovic, D. 1999. Fuzzy logic systems for transportation engineering: the state of the art, Transportation Research Part A: Policy and Practice 33(5): 337-364. http://dx.doi.org/10.1016/S0965-8564(98)00024-X

Vitins, B. J.; Axhausen, K. W. 2009. Optimization of large transport networks using the ant colony heuristic, Computer-Aided Civil and Infrastructure Engineering 24(1): 1-14. http://dx.doi.org/10.1111/j.1467-8667.2008.00569.x

Watling, D. P. 2006. User equilibrium traffic network assignment with stochastic travel times and late arrival penalty, European Journal of Operational Research 175(3): 1539-1556.

Wang, J.; Lu, H.; Peng, H. 2008. System dynamics model of urban transportation system and its application, Journal of Transportation Systems Engineering and Information Technology 8(3): 83-89.

Xu, J. P.; Liu, Y. G. 2008. Multi-objective decision making model under fuzzy random environment and its application to inventory problems, Information Sciences 178: 2899-2914.

Xu, J. P.; Zhou, X. 2011. Fuzzy-like multiple objective decision making. Heidelberg: Springer-Verlag. 440 p.

Xu, J. P.; Liu, Q. 2011. A study on facility location-allocation problem in mixed random and fuzzy environment, Journal of Intelligent Manufacturing 22: 389-398.

Xu, J. P; Zeng, Z. Q. 2011. Applying optimal control model to dynamic equipment allocation problem: case study of concrete-faced rockfill dam construction project, Journal of Construction Engineering and Management ASCE 137(7): 536-550. 
Xu, J. P.; Zheng, H.; Zeng, Z. Q.; Wu, S. Y.; Shen, M. 2012a. Discrete time-cost-environment trade-off problem for large-scale construction systems with multiple modes under fuzzy uncertainty and its application to Jinping-II Hydroelectric Project, International Journal of Project Management 30(8): 950-966. http://dx.doi.org/10.1016/j.ijproman.2012.01.019

Xu, J. P.; Zeng, Z. Q.; Han, B.; Lei, X. 2012b. A dynamic programming-based particle swarm optimization algorithm for an inventory management problem under uncertainty, Engineering Optimization 45(7): 851-880. http://dx.doi.org/10.1080/0305215X.2012.709514
Xu, J. P.; Tu, Y.; Zeng, Z. Q. 2012c. Bi-level optimization of regional water resources allocation problem under fuzzy random environment, Journal of Water Resources Planning and Management 139(3): 246-264.

http://dx.doi.org/10.1061/(ASCE)WR.1943-5452.0000248

Yapicioglu, H.; Smith, A. E.; Dozier, G. 2007.Solving the semidesirable facility location problem using bi-objective particle swarm, European Journal of Operational Research 177: 733-749.

Zadeh, L. A. 1965. Fuzzy sets, Information and Control 8: 338-353.

Jiuping XU. He obtained his PhD in Applied Mathematics from Tsinghua University, and PhD in Physical Chemistry from Sichuan University, in 1995 and 1999, respectively. He is a Distinguished Prof. of the "Chang Jiang Scholars Program" of the Ministry of Education of the P. R. China, the Dean of the Business School of Sichuan University. He was appointed a Lifetime Academician of the International Academy of Systems and Cybernetic Sciences in 2010. He is the President of the International Society for Management Science and Engineering Management, and the Vice-President of The Systems Engineering Society of China, Vice-President of the Chinese Society of Optimization \& Overall Planning and Economical Mathematics, the Editor in Chief of The International Journal of Management Science and Engineering Management. He has published more than 50 official books and 400 journal papers.

Cuiying FENG. She is a PhD candidate of Management Science and Engineering, Sichuan University, P. R. China. Her research interests include transportation planning problem, supply management optimization, construction engineering and management, scheduling and operations management. 